\title{
A Künneth formula in topological homology and its applications to the simplicial cohomology of $\ell^{1}\left(\mathbb{Z}_{+}^{k}\right)$
}

by

\author{
F. Gourdeau (Québec), Z. A. Lykova (Newcastle upon Tyne) \\ and M. C. White (Newcastle upon Tyne)
}

\begin{abstract}
We establish a Künneth formula for some chain complexes in the categories of Fréchet and Banach spaces. We consider a complex $\mathcal{X}$ of Banach spaces and continuous boundary maps $d_{n}$ with closed ranges and prove that $H^{n}\left(\mathcal{X}^{\prime}\right) \cong H_{n}(\mathcal{X})^{\prime}$, where $H_{n}(\mathcal{X})^{\prime}$ is the dual space of the homology group of $\mathcal{X}$ and $H^{n}\left(\mathcal{X}^{\prime}\right)$ is the cohomology group of the dual complex $\mathcal{X}^{\prime}$. A Künneth formula for chain complexes of nuclear Fréchet spaces and continuous boundary maps with closed ranges is also obtained. This enables us to describe explicitly the simplicial cohomology groups $\mathcal{H}^{n}\left(\ell^{1}\left(\mathbb{Z}_{+}^{k}\right), \ell^{1}\left(\mathbb{Z}_{+}^{k}\right)^{\prime}\right)$ and homology groups $\mathcal{H}_{n}\left(\ell^{1}\left(\mathbb{Z}_{+}^{k}\right), \ell^{1}\left(\mathbb{Z}_{+}^{k}\right)\right)$ of the semigroup algebra $\ell^{1}\left(\mathbb{Z}_{+}^{k}\right)$.
\end{abstract}

1. Introduction. One of the most difficult problems in the homology of topological algebras is the calculation of the Hochschild homology and cohomology of algebras; see $[7,5]$. In this paper we consider Banach and Fréchet algebras which can be represented as complete projective tensor products $\mathcal{A} \widehat{\otimes} \mathcal{B}$ of algebras $\mathcal{A}$ and $\mathcal{B}$, and obtain the descriptions of the Hochschild homology $\mathcal{H}_{n}(\mathcal{A} \widehat{\otimes} \mathcal{B}, X \widehat{\otimes} Y)$ of the algebra $\mathcal{A} \widehat{\otimes} \mathcal{B}$ with the aid of a Künneth formula (Corollary 6.4). For the explicit descriptions of the cohomology groups of $\mathcal{A} \widehat{\otimes} \mathcal{B}$ of Banach algebras $\mathcal{A}$ and $\mathcal{B}$ with coefficients in dual bimodules we use the following result. If either the homology of a chain complex $\mathcal{X}$ of Banach spaces, or the cohomology of the dual complex $\mathcal{X}^{\prime}$ is formed of Banach spaces, then both are Banach spaces and further, the cohomology group $H^{n}\left(\mathcal{X}^{\prime}\right)$ is topologically isomorphic to the dual of the homology group $H_{n}(\mathcal{X})$ (Corollary 4.9).

The main tool in this paper is a Künneth formula for bounded chain complexes $\mathcal{X}$ and $\mathcal{Y}$ of Fréchet spaces and continuous boundary maps with closed ranges. In Theorem 5.2 we prove that, under certain topological con-

2000 Mathematics Subject Classification: Primary 46H20, 46J40, 22E41; Secondary 16E40, 43A20.

Key words and phrases: Hochschild cohomology, simplicial cohomology, Banach algebra, semigroup algebra, nuclear Fréchet algebra. 
ditions, there is a topological isomorphism

$$
\bigoplus_{m+q=n}\left[H_{m}(\mathcal{X}) \widehat{\otimes} H_{q}(\mathcal{Y})\right] \cong H_{n}(\mathcal{X} \widehat{\otimes} \mathcal{Y})
$$

It is important to mention that we obtain the Künneth formula in the category of Fréchet spaces and continuous operators, and so we need the notion of strict flatness (Def. 4.2) to deal with this topological case. Recall that the Hochschild homology and cohomology groups of a Fréchet algebra can also be calculated by using the $\mathbb{C}$-relative homological theory which can be found in [7]. One of the main topological conditions in Theorem 5.2 is the topological purity of some short exact sequences of Fréchet spaces. This condition allows us to deal with the known problem in the category of Fréchet spaces that the projective tensor product of injective continuous linear operators is not necessarily injective. We study properties of topologically pure short exact sequences of Fréchet and Banach spaces in Section 3, and properties of strictly flat and strictly injective Banach spaces in Section 4.

The most interesting result of Section 4 is Theorem 4.8. For a chain complex $\mathcal{X}$ of Banach spaces and continuous boundary maps with closed ranges and for a non-zero strictly injective Banach space $I$, there is an isomorphism of Banach spaces

$$
L\left(H_{n}(\mathcal{X}), I\right) \cong H^{n}(L(\mathcal{X}, I)),
$$

where $L(F, E)$ is the Banach space of continuous operators from the Banach space $F$ to the Banach space $E$.

In Section 6 we show that the Hochschild homology group $\mathcal{H}_{n}(\mathcal{A} \widehat{\otimes} \mathcal{B}$, $X \widehat{\otimes} Y)$ of the projective tensor product of unital Fréchet algebras $\mathcal{A}$ and $\mathcal{B}$ is the homology group of the tensor product of the appropriate Hochschild chain complexes. Under certain topological conditions on Hochschild chain complexes, we show that, up to topological isomorphism,

$$
\mathcal{H}_{n}(\mathcal{A} \widehat{\otimes} \mathcal{B}, X \widehat{\otimes} Y)=\bigoplus_{m+q=n}\left[\mathcal{H}_{m}(\mathcal{A}, X) \widehat{\otimes} \mathcal{H}_{q}(\mathcal{B}, Y)\right]
$$

where $X$ is a Fréchet $\mathcal{A}$-bimodule and $Y$ is a Fréchet $\mathcal{B}$-bimodule.

In Section 7 we apply the above results to the calculation of the simplicial homology $\mathcal{H}_{n}\left(\ell^{1}\left(\mathbb{Z}_{+}^{k}\right), \ell^{1}\left(\mathbb{Z}_{+}^{k}\right)\right)$ and cohomology $\mathcal{H}^{n}\left(\ell^{1}\left(\mathbb{Z}_{+}^{k}\right), \ell^{1}\left(\mathbb{Z}_{+}^{k}\right)^{\prime}\right)$ of the unital semigroup algebra $\ell^{1}\left(\mathbb{Z}_{+}^{k}\right)=\ell^{1}\left(\mathbb{Z}_{+}\right) \widehat{\otimes} \ell^{1}\left(\mathbb{Z}_{+}^{k-1}\right), k>1$. In Theorem 7.5 explicit descriptions of the simplicial homology and cohomology of $\ell^{1}\left(\mathbb{Z}_{+}^{k}\right)$ are given. For example, we prove that

(1) $\mathcal{H}^{n}\left(\ell^{1}\left(\mathbb{Z}_{+}^{k}\right), \ell^{1}\left(\mathbb{Z}_{+}^{k}\right)^{\prime}\right)=0$ if $n>k$,

(2) $\mathcal{H}^{n}\left(\ell^{1}\left(\mathbb{Z}_{+}^{k}\right), \ell^{1}\left(\mathbb{Z}_{+}^{k}\right)^{\prime}\right)=\bigoplus^{\left(\begin{array}{l}k \\ n\end{array}\right)}\left[\left(\mathcal{I}^{\widehat{\otimes}^{n}} \widehat{\otimes} \mathcal{A}^{\widehat{\otimes}^{k-n}}\right)^{\prime}\right]$ if $n \leq k$,

where $\mathcal{A}=\ell^{1}\left(\mathbb{Z}_{+}\right)=\left\{\left(a_{n}\right)_{n=0}^{\infty}: \sum_{n=0}^{\infty}\left|a_{n}\right|<\infty\right\}$ is the unital semigroup algebra with convolution multiplication on $\mathbb{Z}_{+}$and norm $\left\|\left(a_{n}\right)_{n=0}^{\infty}\right\|=\sum_{n=0}^{\infty}\left|a_{n}\right|$, 
and $\mathcal{I}=\ell^{1}(\mathbb{N})$ is the closed ideal of $\ell^{1}\left(\mathbb{Z}_{+}\right)$consisting of those elements with $a_{0}=0$.

2. Definitions and notation. We recall some notation and terminology used in homology theory. These can be found in any textbook on homological algebra, for instance, see MacLane [13] for the pure algebraic case and Helemskiı [7] for the continuous case.

The categories of Fréchet and Banach spaces and continuous linear operators are denoted by $\mathcal{F} r$ and $\mathcal{B}$ an respectively. For a Fréchet algebra $\mathcal{A}$, the category of left Fréchet $\mathcal{A}$-modules is denoted by $\mathcal{A}$-mod and the category of Fréchet $\mathcal{A}$-bimodules is denoted by $\mathcal{A}$-mod- $\mathcal{A}$.

A chain complex $\mathcal{X}$ in $\mathcal{F r}$ (resp. in $\mathcal{B a n}$ ) is a family of Fréchet (resp. Banach) spaces $X_{n}$ and continuous linear maps $d_{n}$ (called boundary maps)

$$
\cdots \stackrel{d_{n-2}}{\longleftarrow} X_{n-1} \stackrel{d_{n-1}}{\longleftarrow} X_{n} \stackrel{d_{n}}{\longleftarrow} X_{n+1} \stackrel{d_{n+1}}{\longleftarrow} X_{n+2} \leftarrow \cdots
$$

such that $\operatorname{Im} d_{n} \subset \operatorname{Ker} d_{n-1}$. The subspace $\operatorname{Im} d_{n}$ of $X_{n}$ is denoted by $B_{n}(\mathcal{X})$ and its elements are called boundaries. The Fréchet (resp. Banach) subspace Ker $d_{n-1}$ of $X_{n}$ is denoted by $Z_{n}(\mathcal{X})$ and its elements are cycles. The homology groups of $\mathcal{X}$ are defined by $H_{n}(\mathcal{X})=Z_{n}(\mathcal{X}) / B_{n}(\mathcal{X})$. As usual, we will often drop the subscript $n$ of $d_{n}$. If there is a need to distinguish between various boundary maps on various chain complexes, we will use subscripts, that is, we will denote the boundary maps on $\mathcal{X}$ by $d_{\mathcal{X}}$. A chain complex $\mathcal{X}$ is called bounded if $X_{n}=\{0\}$ whenever $n$ is less than a certain fixed integer $N \in \mathbb{Z}$.

Throughout, given a chain complex $\mathcal{X}$ in $\mathcal{F} r$ (or in $\mathcal{B}$ an), we will consider the following short exact sequences:

$$
\begin{aligned}
0 & \rightarrow Z_{n}(\mathcal{X}) \stackrel{i_{n}}{\longrightarrow} X_{n} \stackrel{\widetilde{d}_{n-1}}{\longrightarrow} B_{n-1}(\mathcal{X}) \rightarrow 0, \\
0 & \rightarrow B_{n}(\mathcal{X}) \stackrel{j_{n}}{\longrightarrow} Z_{n}(\mathcal{X}) \stackrel{\sigma_{n}}{\longrightarrow} H_{n}(\mathcal{X}) \rightarrow 0,
\end{aligned}
$$

where $i_{n}$ is the natural inclusion map from $Z_{n}(\mathcal{X})$ into $X_{n}, j_{n}$ is the natural inclusion map from $B_{n}(\mathcal{X})$ into $Z_{n}(\mathcal{X}), \widetilde{d}_{n-1}$ is the boundary map seen as mapping into its image, and $\sigma_{n}$ is the quotient map. Here the notation $\widetilde{d}$ is an instance of one we shall use repeatedly: given a continuous linear map $\theta: E \rightarrow F$, the map $\widetilde{\theta}$ is the surjective map $\widetilde{\theta}: E \rightarrow \operatorname{Im} \theta$ defined by $\widetilde{\theta}(t)=\theta(t)$.

The short exact sequence of Fréchet spaces and continuous linear operators $0 \rightarrow Y \stackrel{i}{\rightarrow} Z \stackrel{j}{\rightarrow} W \rightarrow 0$ is called admissible if there exist continuous operators $\beta: Z \rightarrow Y$ and $\alpha: W \rightarrow Z$ such that $\beta \circ i=1_{Y}, j \circ \alpha=1_{W}$ and $i \circ \beta+\alpha \circ j=1_{Z}$. Recall that admissibility is equivalent to the existence of a continuous operator $\beta: Z \rightarrow Y$ such that $\beta \circ i=1_{Y}$. A short exact sequence 
of Fréchet spaces and continuous linear operators $0 \rightarrow Y \stackrel{i}{\rightarrow} Z \stackrel{j}{\rightarrow} W \rightarrow 0$ is weakly admissible if the strong dual short exact sequence is admissible.

Let $\mathcal{K}$ be one of the above categories of Fréchet (or Banach) $\mathcal{A}$-modules and their morphisms. A complex of Fréchet $\mathcal{A}$-modules and their morphisms is called admissible if it splits as a complex of Fréchet spaces [7, III.3.11]. For $Y \in \mathcal{A}$-mod- $\mathcal{A}$ a complex

$$
0 \leftarrow Y \stackrel{\varepsilon}{\leftarrow} P_{0} \stackrel{\phi_{0}}{\longleftarrow} P_{1} \stackrel{\phi_{1}}{\longleftarrow} P_{2} \leftarrow \cdots \quad(0 \leftarrow Y \leftarrow \mathcal{P})
$$

over $Y$ is called a projective resolution in $\mathcal{A}$-mod- $\mathcal{A}$ if it is admissible and all the modules in $\mathcal{P}$ are projective in $\mathcal{A}$-mod- $\mathcal{A}$ [7, Definition III.2.1].

For a unital Fréchet algebra $\mathcal{A}$, the algebra $\mathcal{A}^{\mathrm{e}}=\mathcal{A} \widehat{\otimes} \mathcal{A}^{\mathrm{op}}$ is called the enveloping algebra of $\mathcal{A}$, where $\mathcal{A}^{\text {op }}$ is the opposite algebra of $\mathcal{A}$ with multiplication $a \cdot b=b a$. For $Y, X \in \mathcal{A}$-mod- $\mathcal{A}$, we shall denote by $\operatorname{Tor}_{n}^{\mathcal{A}^{\mathrm{e}}}(X, Y)$ the $n$th homology of the complex $X \widehat{\otimes}_{\mathcal{A}^{\mathrm{e}}} \mathcal{P}$, where $0 \leftarrow Y \leftarrow \mathcal{P}$ is a projective resolution in $\mathcal{A}$-mod- $\mathcal{A}$ [7, Definition III.4.23]. Here $\widehat{\otimes}$ is the projective tensor product of Fréchet (resp. Banach) spaces [3], [7, II.4.1], and $\widehat{\otimes}_{\mathcal{A}}$ is the projective tensor product of left and right Fréchet (resp. Banach) $\mathcal{A}$-modules (see [15]). Note that by $X^{\widehat{\otimes} 0} \widehat{\otimes} Y$ we mean $Y$, by $X^{\widehat{\otimes} 1}$ we mean $X$ and by $X^{\widehat{\otimes} n}$ we mean the $n$-fold projective tensor power $X \widehat{\otimes} \cdots \widehat{\otimes} X$ of $X$.

Given a Fréchet (resp. Banach) space $E$ and a chain complex $(\mathcal{X}, d)$ in $\mathcal{F r}$ (resp. in $\mathcal{B} a n$ ), we can form the chain complex $E \widehat{\otimes} \mathcal{X}$ of the Fréchet (resp. Banach) spaces $E \widehat{\otimes} X_{n}$ and boundary maps $1_{E} \otimes d$. Definitions of the totalization $\operatorname{Tot}(\overline{\mathcal{M}})$ of a bounded bicomplex $\overline{\mathcal{M}}$ and the tensor product $\mathcal{X} \widehat{\otimes} \mathcal{Y}$ of bounded complexes $\mathcal{X}$ and $\mathcal{Y}$ in $\mathcal{F} r$ can be found in [7, Definitions II.5.23-25]. Recall that $\mathcal{X} \widehat{\otimes} \mathcal{Y}:=\operatorname{Tot}(\overline{\mathcal{X} \widehat{\otimes} \mathcal{Y}})$ for a bounded bicomplex $\overline{\mathcal{X} \widehat{\otimes} \mathcal{Y}}$.

Finally, throughout the paper $1_{X}: X \rightarrow X$ denotes the identity operator and $\cong$ denotes an isomorphism of Fréchet (or Banach) spaces, as appropriate. Many of our proofs depend on the Open Mapping Theorem for Fréchet spaces; one can find it in [4, Theorem 6.4.5].

\section{Topologically pure extensions of Fréchet spaces and homology groups}

Definition 3.1. A short exact sequence of Fréchet (resp. Banach) spaces and continuous linear operators

$$
0 \rightarrow Y \stackrel{i}{\rightarrow} Z \stackrel{j}{\rightarrow} W \rightarrow 0
$$

is called topologically pure in $\mathcal{F} r$ (resp. in $\mathcal{B}$ an) if for every $X \in \mathcal{F} r$ (resp. $X \in \mathcal{B}$ an) the sequence

is exact.

$$
0 \rightarrow X \widehat{\otimes} Y \stackrel{1_{X} \otimes i}{\longrightarrow} X \widehat{\otimes} Z \stackrel{1_{X} \otimes j}{\longrightarrow} X \widehat{\otimes} W \rightarrow 0
$$


An extension of Fréchet algebras is called topologically pure if the underlying short exact sequence is topologically pure in $\mathcal{F} r$. One can find the definition and properties of topologically pure extensions in [2] and [12] for the Fréchet case, while the Banach case is treated in [11]. Recall that extensions of Fréchet spaces which satisfy one of the following conditions are topologically pure:

(i) admissible or weakly admissible extensions;

(ii) extensions of nuclear Fréchet spaces ([5, Theorems A.1.6 and A.1.5]);

(iii) extensions of Fréchet algebras such that $Y$ has a left or right bounded approximate identity.

The reason for the introduction of topologically pure extensions of Fréchet algebras is that they allow one to circumvent the known problem that the projective tensor product of injective continuous linear operators is not necessarily injective, as well as ensure that $\left(1_{X} \otimes i\right)(X \widehat{\otimes} Y)$ is closed in $X \widehat{\otimes} Z$.

LEMmA 3.2. A short exact sequence of Banach spaces and continuous linear operators

$$
0 \rightarrow Y \stackrel{i}{\rightarrow} Z \stackrel{j}{\rightarrow} W \rightarrow 0
$$

is weakly admissible in $\mathcal{B}$ an if and only if it is topologically pure in $\mathcal{B} a n$.

Proof. This follows from [3, II.1.8f and Remark after II.1.9]. See also [11, Lemma 3.3] for a proof that a weakly admissible short exact sequence is necessarily topologically pure.

The following result for the pure algebraic case can be found in [13, Lemma V.10.3].

Proposition 3.3. Let $G$ be a Fréchet (resp. Banach) space, let $\mathcal{X}$ be a chain complex in $\mathcal{F r}$ (resp. in $\mathcal{B}$ an) such that all boundary maps d have closed range, let $G \widehat{\otimes} \mathcal{X}$ be the chain complex with boundary maps $1_{G} \otimes d$, and let $n \in \mathbb{Z}$. Suppose that, for $k=n-1$ and for $k=n$, the exact sequences of Fréchet (resp. Banach) spaces

$$
\begin{aligned}
& 0 \rightarrow Z_{k}(\mathcal{X}) \stackrel{i_{k}}{\longrightarrow} X_{k} \stackrel{\widetilde{d}_{k-1}}{\longrightarrow} B_{k-1}(\mathcal{X}) \rightarrow 0, \\
& 0 \rightarrow B_{k}(\mathcal{X}) \stackrel{j_{k}}{\longrightarrow} Z_{k}(\mathcal{X}) \stackrel{\sigma_{k}}{\longrightarrow} H_{k}(\mathcal{X}) \rightarrow 0
\end{aligned}
$$

are topologically pure in $\mathcal{F r}$ (resp. in $\mathcal{B}$ an). Then the natural inclusions induce topological isomorphisms:

(i) $G \widehat{\otimes} Z_{n}(\mathcal{X}) \cong Z_{n}(G \widehat{\otimes} \mathcal{X})$,

(ii) $G \widehat{\otimes} B_{n}(\mathcal{X}) \cong B_{n}(G \widehat{\otimes} \mathcal{X})$,

(iii) $G \widehat{\otimes} H_{n}(\mathcal{X}) \cong G \widehat{\otimes} Z_{n}(\mathcal{X}) /\left(1_{G} \otimes j_{n}\right)\left(G \widehat{\otimes} B_{n}(\mathcal{X})\right)$

$$
\cong Z_{n}(G \widehat{\otimes} \mathcal{X}) / B_{n}(G \widehat{\otimes} \mathcal{X})=H_{n}(G \widehat{\otimes} \mathcal{X}) \text {. }
$$

In particular, the boundary map $1_{G} \otimes d_{n}$ also has closed range. 
Proof. For $k=n-1$ or $k=n$, since the sequence (3) is topologically pure, the sequence

$$
0 \rightarrow G \widehat{\otimes} B_{k}(\mathcal{X}) \stackrel{1_{G} \otimes j_{k}}{\longrightarrow} G \widehat{\otimes} Z_{k}(\mathcal{X}) \stackrel{1_{G} \otimes \sigma_{k}}{\longrightarrow} G \widehat{\otimes} H_{k}(\mathcal{X}) \rightarrow 0
$$

is exact. Therefore $1_{G} \otimes j_{k}$ is injective, $\operatorname{Im}\left(1_{G} \otimes j_{k}\right)$ is closed and

$$
G \widehat{\otimes} H_{k}(\mathcal{X}) \cong G \widehat{\otimes} Z_{k}(\mathcal{X}) /\left(1_{G} \otimes j_{k}\right)\left(G \widehat{\otimes} B_{k}(\mathcal{X})\right) .
$$

By the Open Mapping Theorem, $\left(1_{G} \otimes j_{k}\right)\left(G \widehat{\otimes} B_{k}(\mathcal{X})\right) \cong G \widehat{\otimes} B_{k}(\mathcal{X})$, so that $1_{G} \otimes j_{k}: G \widehat{\otimes} B_{k}(\mathcal{X}) \rightarrow G \widehat{\otimes} Z_{k}(\mathcal{X})$ identifies $G \widehat{\otimes} B_{k}(\mathcal{X})$ as a closed subspace of $G \widehat{\otimes} Z_{k}(\mathcal{X})$.

For $k=n-1$ or $k=n$, since the sequence (2) is topologically pure, the sequence

$$
0 \rightarrow G \widehat{\otimes} Z_{k}(\mathcal{X}) \stackrel{1_{G} \otimes i_{k}}{\longrightarrow} G \widehat{\otimes} X_{k} \stackrel{1_{G} \otimes \tilde{d}_{k-1}}{\longrightarrow} G \widehat{\otimes} B_{k-1}(\mathcal{X}) \rightarrow 0
$$

is exact. Therefore $1_{G} \otimes i_{k}$ is injective and

$$
\operatorname{Im}\left(1_{G} \otimes i_{k}\right)=\operatorname{Ker}\left(1_{G} \otimes \widetilde{d}_{k-1}\right) .
$$

Hence, by the Open Mapping Theorem,

$$
G \widehat{\otimes} Z_{k}(\mathcal{X}) \cong \operatorname{Ker}\left(1_{G} \otimes \widetilde{d}_{k-1}\right) .
$$

For $k=n-1$ or $k=n$, consider the commutative diagram

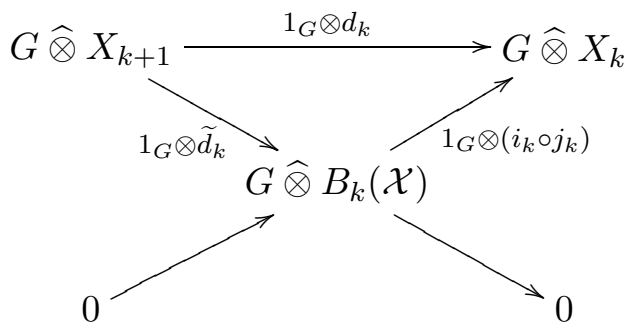

Since a projective tensor product of surjective continuous linear operators is surjective, $1_{G} \otimes \widetilde{d}_{k}$ is surjective and

$$
\left(1_{G} \otimes \widetilde{d}_{k}\right)\left(G \widehat{\otimes} X_{k+1}\right)=G \widehat{\otimes} B_{k}(\mathcal{X}) .
$$

Therefore, since the diagram is commutative, $1_{G} \otimes d_{k}$ has the same image as $1_{G} \otimes\left(i_{k} \circ j_{k}\right)$. We have proved that $1_{G} \otimes\left(i_{k} \circ j_{k}\right)$ is injective. As $\operatorname{Im}\left(1_{G} \otimes\left(i_{k} \circ j_{k}\right)\right)$ is closed, by the Open Mapping Theorem,

$$
\operatorname{Im}\left(1_{G} \otimes d_{k}\right)=\operatorname{Im}\left(1_{G} \otimes\left(i_{k} \circ j_{k}\right)\right) \cong G \widehat{\otimes} B_{k}(\mathcal{X}) .
$$

Hence

$$
B_{k}(G \widehat{\otimes} \mathcal{X}) \cong G \widehat{\otimes} B_{k}(\mathcal{X}),
$$

and this proves (ii). Note also that $\operatorname{Im}\left(1_{G} \otimes d_{k}\right) \cong \operatorname{Im}\left(1_{G} \otimes \widetilde{d}_{k}\right)$. 
We have proved that $1_{G} \otimes\left(i_{k} \circ j_{k}\right)$ is injective, so that

$$
\operatorname{Ker}\left(1_{G} \otimes d_{k}\right)=\operatorname{Ker}\left(1_{G} \otimes \widetilde{d}_{k}\right) .
$$

Therefore, by (5),

$$
Z_{n}(G \widehat{\otimes} \mathcal{X})=\operatorname{Ker}\left(1_{G} \otimes d_{n-1}\right)=\operatorname{Ker}\left(1_{G} \otimes \widetilde{d}_{n-1}\right) \cong G \widehat{\otimes} Z_{n}(\mathcal{X}) .
$$

This proves (i).

Finally, by (7), (6) and (4), we have the following topological isomorphisms:

$$
\begin{aligned}
H_{n}(G \widehat{\otimes} \mathcal{X}) & =Z_{n}(G \widehat{\otimes} \mathcal{X}) / B_{n}(G \widehat{\otimes} \mathcal{X}) \\
& \cong G \widehat{\otimes} Z_{n}(\mathcal{X}) /\left(1_{G} \otimes j_{n}\right)\left(G \widehat{\otimes} B_{n}(\mathcal{X})\right) \cong G \widehat{\otimes} H_{n}(\mathcal{X}) .
\end{aligned}
$$

4. Strictly flat Fréchet spaces and homology groups. The following two definitions are equivalent to those given in [7, Chapter VII].

Definition 4.1. A Fréchet (resp. Banach) space $I$ is strictly injective if for every pair of Fréchet (resp. Banach) spaces $E$ and $F$, for every injective continuous linear operator $i: E \rightarrow F$ with closed range and for every continuous linear operator $\theta: E \rightarrow I$, there is a continuous linear operator $\vartheta: F \rightarrow I$ such that $\vartheta \circ i=\theta$.

Definition 4.2. A Fréchet (resp. Banach) space $G$ is strictly flat if for every short exact sequence of Fréchet (resp. Banach) spaces and continuous linear operators $0 \rightarrow X \rightarrow Y \rightarrow Z \rightarrow 0$, the short exact sequence

$$
0 \rightarrow G \widehat{\otimes} X \rightarrow G \widehat{\otimes} Y \rightarrow G \widehat{\otimes} Z \rightarrow 0
$$

is also exact.

Lemma 4.3. Let

$$
0 \rightarrow Y \stackrel{i}{\rightarrow} Z \stackrel{j}{\rightarrow} W \rightarrow 0
$$

be a short exact sequence of Banach spaces and continuous linear operators. Suppose $W$ is strictly flat in $\mathcal{B a n}$. Then the sequence (8) is weakly admissible and therefore is topologically pure in $\mathcal{B}$ an.

Proof. Since $j$ is surjective, the dual map $j^{*}: W^{*} \rightarrow Z^{*}$ is injective and has a closed range. By [7, Theorem VII.1.14], $W^{*}$ is strictly injective. Therefore, for $j^{*}: W^{*} \rightarrow Z^{*}$ and the identity operator $1_{W^{*}}: W^{*} \rightarrow W^{*}$, there is a continuous linear operator $\theta: Z^{*} \rightarrow W^{*}$ such that $\theta \circ j^{*}=1_{W^{*}}$. Thus the sequence (8) is weakly admissible and, by [11, Lemma 3.3], is topologically pure.

Lemma 4.4. Let $\theta: E \rightarrow F$ be a continuous linear map with closed range between Fréchet (resp. Banach) spaces and let $G$ be a strictly flat Fréchet 
(resp. Banach) space. Let the maps $\kappa$ and $q$ be defined by the exact sequence

$$
0 \rightarrow \operatorname{Ker} \theta \stackrel{\kappa}{\rightarrow} E \stackrel{\theta}{\rightarrow} F \stackrel{q}{\rightarrow} F / \operatorname{Im} \theta \rightarrow 0 .
$$

Then

(i) the sequence

$0 \rightarrow G \widehat{\otimes} \operatorname{Ker} \theta \stackrel{1_{G} \otimes \kappa}{\longrightarrow} G \widehat{\otimes} E \stackrel{1_{G} \otimes \theta}{\longrightarrow} G \widehat{\otimes} F \stackrel{1_{G} \otimes q}{\longrightarrow} G \widehat{\otimes}(F / \operatorname{Im} \theta) \rightarrow 0$ is exact,

(ii) $G \widehat{\otimes} \operatorname{Im} \theta \cong \operatorname{Im}\left(1_{G} \otimes i\right)=\operatorname{Ker}\left(1_{G} \otimes q\right)=\operatorname{Im}\left(1_{G} \otimes \theta\right)$,

(iii) $\operatorname{Ker}\left(1_{G} \otimes \theta\right)=\operatorname{Ker}\left(1_{G} \otimes \widetilde{\theta}\right)=\operatorname{Im}\left(1_{G} \otimes \kappa\right) \cong G \widehat{\otimes} \operatorname{Im} \kappa=G \widehat{\otimes} \operatorname{Ker} \theta$, where $i: \operatorname{Im} \theta \rightarrow F$ is the natural inclusion.

Proof. Consider the commutative diagram

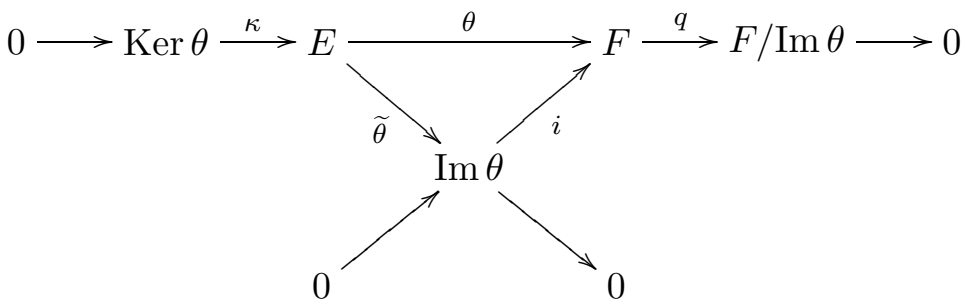

The extra term $\operatorname{Im} \theta$ produces two short exact sequences. Tensoring this diagram with $G$ maintains exactness of the short exact sequences as $G$ is strictly flat:

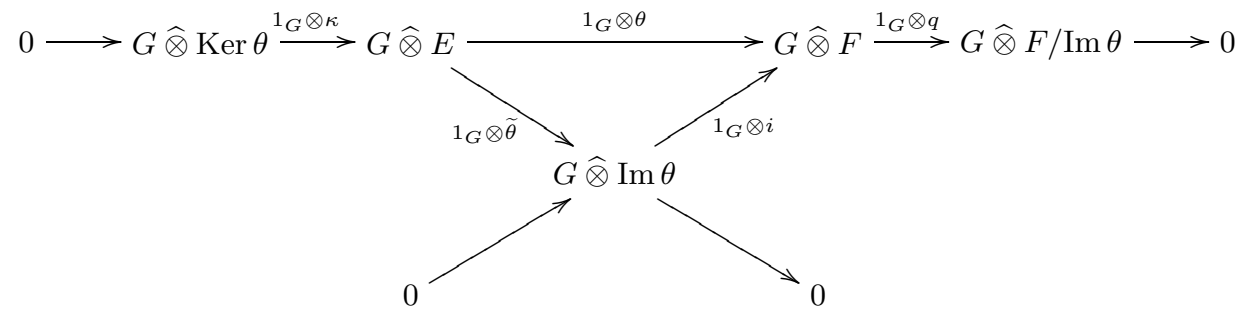

It can be argued directly that the long sequence is exact, but this also follows from [7, Definition VII.I.3 and Proposition VII.1.4]. Either way, the sequence (9) is exact.

We now observe that $1_{G} \otimes \widetilde{\theta}$ is surjective and since the diagram is commutative, $1_{G} \otimes \theta$ has the same image as $1_{G} \otimes i$. By the Open Mapping Theorem, $G \widehat{\otimes} \operatorname{Im} \theta \cong \operatorname{Im}\left(1_{G} \otimes i\right)$. By the exactness of

$$
0 \rightarrow G \widehat{\otimes} \operatorname{Im} \theta \stackrel{1_{G} \otimes i}{\longrightarrow} G \widehat{\otimes} F \stackrel{1_{G} \otimes q}{\longrightarrow} G \widehat{\otimes}(F / \operatorname{Im} \theta) \rightarrow 0,
$$

$\operatorname{Im}\left(1_{G} \otimes i\right)=\operatorname{Ker}\left(1_{G} \otimes q\right)$. Hence we have

$$
G \widehat{\otimes} \operatorname{Im} \theta \cong \operatorname{Im}\left(1_{G} \otimes i\right)=\operatorname{Im}\left(1_{G} \otimes \theta\right)=\operatorname{Ker}\left(1_{G} \otimes q\right) .
$$


Next observe that $1_{G} \otimes i$ is injective, so that

$\operatorname{Ker}\left(1_{G} \otimes \theta\right)=\operatorname{Ker}\left(1_{G} \otimes \widetilde{\theta}\right)=\operatorname{Im}\left(1_{G} \otimes \kappa\right) \cong G \widehat{\otimes} \operatorname{Im} \kappa=G \widehat{\otimes} \operatorname{Ker} \theta$.

Proposition 4.5. Let $G$ be a strictly flat Fréchet (resp. Banach) space, let $\mathcal{X}$ be a chain complex in $\mathcal{F} r$ (resp. in $\mathcal{B}$ an) such that all boundary maps $d_{n}$ have closed range, and let $G \widehat{\otimes} \mathcal{X}$ be the chain complex with boundary maps $1_{G} \otimes d_{n}$. Then, for all $n$, the natural inclusions induce topological isomorphisms:

(i) $G \widehat{\otimes} Z_{n}(\mathcal{X}) \cong Z_{n}(G \widehat{\otimes} \mathcal{X})$,

(ii) $G \widehat{\otimes} B_{n}(\mathcal{X}) \cong B_{n}(G \widehat{\otimes} \mathcal{X})$,

(iii) $G \widehat{\otimes} H_{n}(\mathcal{X}) \cong G \widehat{\otimes} Z_{n}(\mathcal{X}) /\left(1_{G} \otimes j_{n}\right)\left(G \widehat{\otimes} B_{n}(\mathcal{X})\right)$

$$
\cong Z_{n}(G \widehat{\otimes} \mathcal{X}) / B_{n}(G \widehat{\otimes} \mathcal{X})=H_{n}(G \widehat{\otimes} \mathcal{X}) .
$$

In particular, all boundary maps $1_{G} \otimes d_{n}$ also have closed range.

Proof. The first statement follows directly from Lemma 4.4 applied to the map $d_{n-1}$, since

$$
Z_{n}(G \widehat{\otimes} \mathcal{X})=\operatorname{Ker}\left(1_{G} \otimes d_{n-1}\right) \cong G \widehat{\otimes} \operatorname{Ker} d_{n-1}=G \widehat{\otimes} Z_{n}(\mathcal{X}) .
$$

The second statement follows from the same Lemma 4.4 applied to the map $d_{n}$, since

$$
B_{n}(G \widehat{\otimes} \mathcal{X})=\operatorname{Im}\left(1_{G} \otimes d_{n}\right) \cong G \widehat{\otimes} \operatorname{Im} d_{n}=G \widehat{\otimes} B_{n}(\mathcal{X}) .
$$

Lemma 4.4 also shows that $1_{G} \otimes d_{n}$ has closed range.

Since $G$ is strictly flat, for the short exact sequence of Fréchet (resp. Banach) spaces

$$
0 \rightarrow B_{n}(\mathcal{X}) \stackrel{j_{n}}{\longrightarrow} Z_{n}(\mathcal{X}) \stackrel{\sigma_{n}}{\longrightarrow} H_{n}(\mathcal{X}) \rightarrow 0
$$

the sequence

$$
0 \rightarrow G \widehat{\otimes} B_{n}(\mathcal{X}) \stackrel{1_{G} \otimes j_{n}}{\longrightarrow} G \widehat{\otimes} Z_{n}(\mathcal{X}) \stackrel{1_{G} \otimes \sigma_{n}}{\longrightarrow} G \widehat{\otimes} H_{n}(\mathcal{X}) \rightarrow 0
$$

is also exact, and therefore $1_{G} \otimes j_{n}$ is injective, $\operatorname{Im}\left(1_{G} \otimes j_{n}\right)=\operatorname{Ker}\left(1_{G} \otimes \sigma_{n}\right)$ and

$$
G \widehat{\otimes} \mathcal{H}_{n}(\mathcal{X}) \cong\left(G \widehat{\otimes} Z_{n}(\mathcal{X})\right) /\left(1_{G} \otimes j_{n}\right)\left(G \widehat{\otimes} B_{n}(\mathcal{X})\right) .
$$

By the Open Mapping Theorem, $1_{G} \otimes j_{n}: G \widehat{\otimes} B_{n}(\mathcal{X}) \rightarrow G \widehat{\otimes} Z_{n}(\mathcal{X})$ identifies $G \widehat{\otimes} B_{n}(\mathcal{X})$ as a closed subspace of $G \widehat{\otimes} Z_{n}(\mathcal{X})$. Hence, by (i) and (ii),

$$
\begin{aligned}
G \widehat{\otimes} H_{n}(\mathcal{X}) & \cong G \widehat{\otimes} Z_{n}(\mathcal{X}) /\left(1_{G} \otimes j_{n}\right)\left(G \widehat{\otimes} B_{n}(\mathcal{X})\right) \\
& \cong Z_{n}(G \widehat{\otimes} \mathcal{X}) / B_{n}(G \widehat{\otimes} \mathcal{X})=H_{n}(G \widehat{\otimes} \mathcal{X}) .
\end{aligned}
$$

Let us denote the Banach space of continuous linear operators between Banach spaces $X$ and $Y$ by $L(X, Y)$ and, for $\phi: X \rightarrow W$, let $h(\phi)$ : $L(W, Y) \rightarrow L(X, Y): \gamma \mapsto \gamma \circ \phi$. The next lemma is analogous to Lemma 4.4. 
Lemma 4.6. Let $\theta: E \rightarrow F$ be a continuous linear map with closed range between Banach spaces and let $I$ be a strictly injective Banach space. Let the maps $\kappa$ and $q$ be defined by the exact sequence

$$
0 \rightarrow \operatorname{Ker} \theta \stackrel{\kappa}{\longrightarrow} E \stackrel{\theta}{\longrightarrow} F \stackrel{q}{\rightarrow} F / \operatorname{Im} \theta \rightarrow 0 .
$$

Then

(i) the sequence

$$
\begin{aligned}
& 0 \leftarrow L(\operatorname{Ker} \theta, I) \stackrel{h(\kappa)}{\longleftarrow} L(E, I) \stackrel{h(\theta)}{\longleftarrow} L(F, I) \stackrel{h(q)}{\longleftarrow} L(F / \operatorname{Im} \theta, I) \leftarrow 0 \\
& \text { is exact, }
\end{aligned}
$$

(ii) $L(\operatorname{Im} \theta, I) \cong \operatorname{Ker} h(\kappa)=\operatorname{Im} h(\widetilde{\theta})=\operatorname{Im} h(\theta)$,

(iii) $L(F / \operatorname{Im} \theta, I) \cong \operatorname{Im} h(q)=\operatorname{Ker} h(i)=\operatorname{Ker} h(\theta)$, where $i: \operatorname{Im} \theta \rightarrow F$ is the natural inclusion.

Proof. We consider the commutative diagram

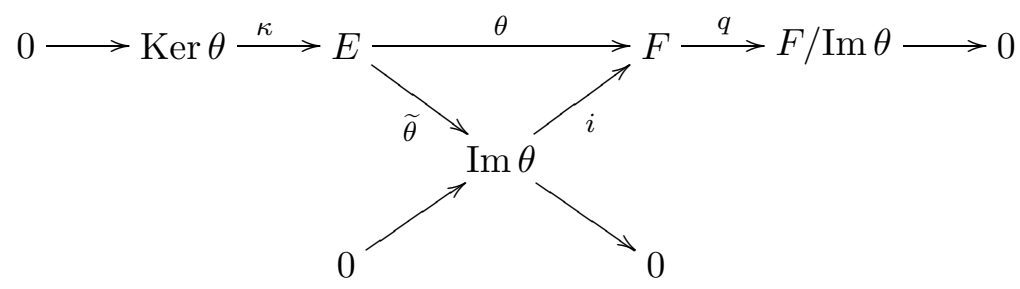

Since $I$ is strictly injective, by [7, Proposition VII.1.12] the extra term $L(\operatorname{Im} \theta, I)$ produces two short exact sequences:

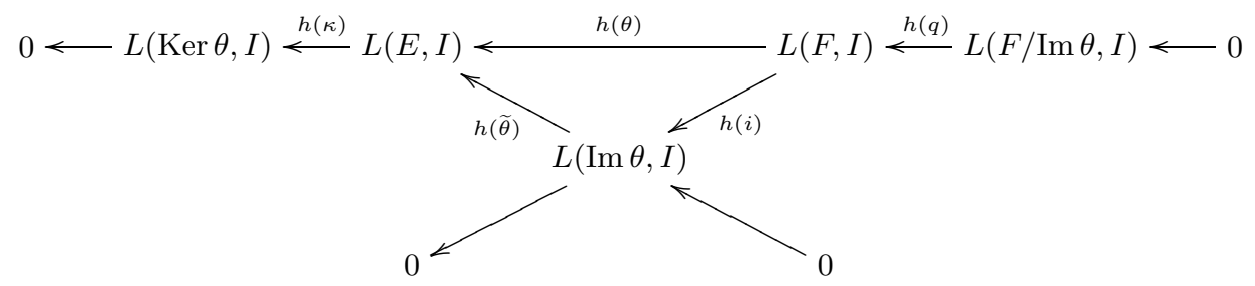

We now observe that $h(i)$ is surjective and so $h(\theta)$ has the same image as $h(\widetilde{\theta})$, which by exactness is the kernel of $h(\kappa)$. Hence we have

$$
L(\operatorname{Im} \theta, I) \cong \operatorname{Ker} h(\kappa)=\operatorname{Im} h(\widetilde{\theta})=\operatorname{Im} h(\theta) .
$$

Next observe that $h(\widetilde{\theta})$ is injective, so that $\operatorname{Ker} h(i)=\operatorname{Ker} h(\theta)$. By the Open Mapping Theorem,

$$
L(F / \operatorname{Im} \theta, I) \cong \operatorname{Im} h(q)=\operatorname{Ker} h(i)=\operatorname{Ker} h(\theta) .
$$

It follows from Lemma 4.6(ii) that if a morphism of Banach spaces $\theta$ : $E \rightarrow F$ has closed range, then so does $h(\theta): L(F, I) \rightarrow L(E, I)$ for a strictly 
injective module $I$. The converse of this result also holds when $I \neq\{0\}$ and will be used in Theorem 4.8. In fact, we have the following lemma.

Lemma 4.7. Let $\theta: E \rightarrow F$ be a continuous linear map between Banach spaces, let $W$ be a non-trivial Banach space and let $h(\theta): L(F, W) \rightarrow$ $L(E, W)$ be the induced map. If $h(\theta)$ has closed range, then so does $\theta$.

Proof. Fix a non-zero $w_{0} \in W$ and $\lambda \in W^{\prime}$ such that $\lambda\left(w_{0}\right)=1$. Let $\lambda_{F}: L(F, W) \rightarrow F^{\prime}$ be defined by $\lambda_{F}(T)(f)=\lambda(T(f))$, where $T \in L(F, W)$, $f \in F$, with a similar definition for $\lambda_{E}: L(E, W) \rightarrow E^{\prime}$. Consider the following commutative diagram, where $\theta^{*}: F^{\prime} \rightarrow E^{\prime}$ is the dual map:

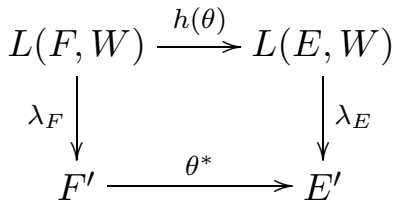

We shall prove that $\theta^{*}$ has closed range, which is equivalent to $\theta$ having closed range (see, for instance, [8, Lemma 1.1]).

The continuous linear map $\beta_{F}: F^{\prime} \rightarrow L(F, W)$ defined by $\beta_{F}(f)(y)$ $=f(y) w_{0}$, for $y \in F$ and $f \in F^{\prime}$, is a right inverse to $\lambda_{F}$. Similarly, $\beta_{E}$ : $E^{\prime} \rightarrow L(E, W)$ is a right inverse to $\lambda_{E}$. Now consider a sequence $g_{n}=\theta^{*}\left(f_{n}\right), n \in \mathbb{N}$, with $g_{n}$ tending to $g_{0}$ in norm in $E^{\prime}$. Then $h(\theta)\left[\beta_{F}\left(f_{n}\right)\right]=\beta_{E}\left(g_{n}\right)$. Therefore $\beta_{E}\left(g_{n}\right)$ is a sequence in $\operatorname{Im} h(\theta)$ which tends to $\beta_{E}\left(g_{0}\right)$. As $h(\theta)$ has closed range, there exists $T_{0} \in L(F, W)$ such that $h(\theta)\left(T_{0}\right)=\beta_{E}\left(g_{0}\right)$. As the diagram commutes, we get $\theta^{*}\left(\lambda_{F}\left(T_{0}\right)\right)=$ $\lambda_{E} \circ \beta_{E}\left(g_{0}\right)=g_{0}$ and $\theta^{*}$ has closed range.

A corollary of the following theorem will allow us to determine the cohomology groups knowing the homology groups, provided they are Banach spaces.

TheORem 4.8. Let $\mathcal{X}$ be a chain complex in $\mathcal{B}$ an and let $I$ be a nontrivial strictly injective Banach space. Let the cochain complex $L(\mathcal{X}, I)$ consist of the Banach spaces $L\left(X_{n}, I\right)$ with induced boundary maps $h\left(d_{n}\right)$. If either $h\left(d_{n}\right)$ has closed range for all $n$, or $d_{n}$ has closed range for all $n$, then

$$
L\left(H_{n}(\mathcal{X}), I\right) \cong H^{n}(L(\mathcal{X}, I)),
$$

where $H^{n}(L(\mathcal{X}, I))=\operatorname{Ker} h\left(d_{n}\right) / \operatorname{Im} h\left(d_{n-1}\right)$ and the isomorphism is of Banach spaces.

Proof. If either $h\left(d_{n}\right)$ has closed range for all $n$ or $d_{n}$ has closed range for all $n$, then Lemma 4.6 (ii) or Lemma 4.7 implies that $d_{n}$ and $h\left(d_{n}\right)$ have closed range for all $n$. We consider the following commutative diagram as in 
[13, Lemma V.10.3]:

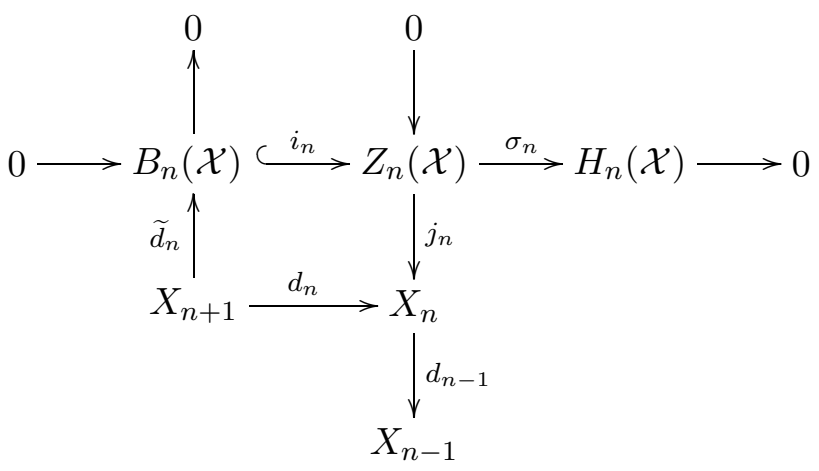

Here again all the maps have closed range.

We form a new diagram by taking continuous linear operators into $I$, adding the kernel $Z^{n}(L(\mathcal{X}, I))=\operatorname{Ker} h\left(d_{n}\right)$ of $h\left(d_{n}\right)$ and the image $B^{n}(L(\mathcal{X}, I))=\operatorname{Im} h\left(d_{n-1}\right)$ of $h\left(d_{n-1}\right)$ :

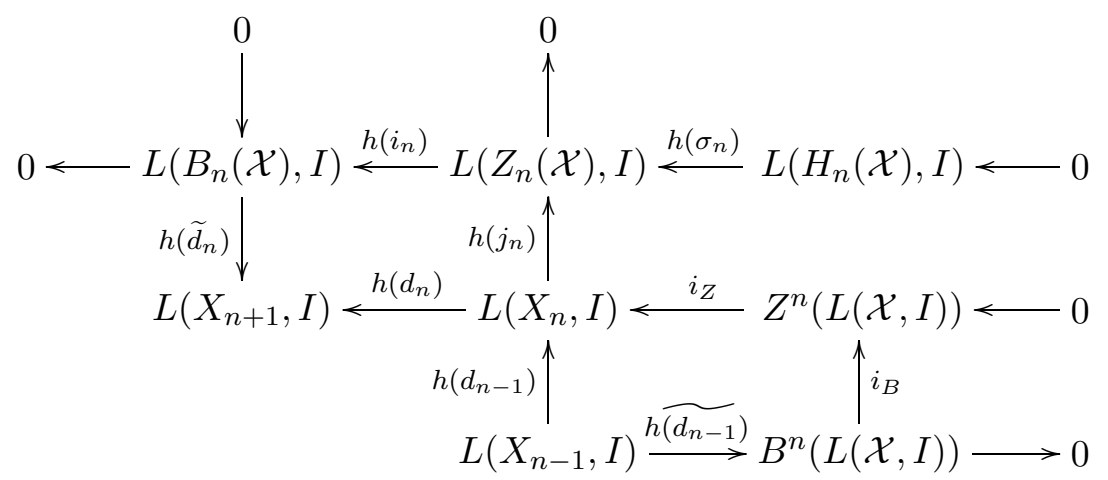

where $\widetilde{h\left(d_{n-1}\right)}: L\left(X_{n-1}, I\right) \rightarrow \operatorname{Im} h\left(d_{n-1}\right): \gamma \mapsto\left[h\left(d_{n-1}\right)\right](\gamma)=\gamma \circ d_{n-1}$. This diagram commutes and has exact rows and columns. Exactness follows from Lemma 4.6 for the first line; from the definition of $Z^{n}(L(\mathcal{X}, I))$ for the second line; and from Lemma 4.6 for the first and second column. Commutativity only needs to be checked for the square involving the two added terms, namely $Z^{n}(L(\mathcal{X}, I))$ and $B^{n}(L(\mathcal{X}, I))$, and this is obvious. The fact that $B^{n}(L(\mathcal{X}, I))$ is closed follows from the exactness of

$$
L\left(X_{n-1}, I\right) \stackrel{h\left(d_{n-1}\right)}{\longrightarrow} L\left(X_{n}, I\right) \stackrel{h\left(j_{n}\right)}{\longrightarrow} L\left(Z_{n}(\mathcal{X}), I\right) \rightarrow 0 .
$$

Therefore this diagram is one of Banach spaces and maps with closed range.

Let us define a map $\varphi: Z^{n}(L(\mathcal{X}, I)) \rightarrow L\left(H_{n}(\mathcal{X}), I\right)$ by the formula

$$
\varphi={\widetilde{h\left(\sigma_{n}\right)}}^{-1} \circ h\left(j_{n}\right) \circ i_{Z},
$$

where ${\widetilde{h\left(\sigma_{n}\right)}}^{-1}$ is the inverse of the topological isomorphism $\widetilde{h\left(\sigma_{n}\right)}$ : 
$L\left(H_{n}(\mathcal{X}), I\right) \rightarrow \operatorname{Im} h\left(\sigma_{n}\right): \gamma \mapsto h\left(\sigma_{n}\right)(\gamma)$. It is now a standard diagram chasing argument to show that $\varphi$ is well defined and surjective. Let us give this argument. An element $z \in Z^{n}(L(\mathcal{X}, I))$ is sent by $h\left(d_{n}\right) \circ i_{Z}$ to 0 in $L\left(X_{n+1}, I\right)$ and therefore, since $h\left(\widetilde{d}_{n}\right)$ is injective, $\left[h\left(i_{n}\right) \circ h\left(j_{n}\right) \circ i_{Z}\right](z)=0$. Hence the element $\left[h\left(j_{n}\right) \circ i_{Z}\right](z)$ of $L\left(Z_{n}(\mathcal{X}), I\right)$ belongs to $\operatorname{Ker} h\left(i_{n}\right)=\operatorname{Im} h\left(\sigma_{n}\right)$, by exactness of the first line of the diagram. Thus $\varphi$ is a well defined continuous linear operator. To show that this map is surjective, starting with $v \in L\left(H_{n}(\mathcal{X}), I\right)$, we get $u=h\left(\sigma_{n}\right)(v) \in L\left(Z_{n}(\mathcal{X}), I\right)$, and, since $h\left(j_{n}\right)$ is surjective, there is $t \in L\left(X_{n}, I\right)$ such that $h\left(j_{n}\right)(t)=u$. It is easy to see that $t \in \operatorname{Ker} h\left(d_{n}\right)$ and therefore it lifts uniquely to $z \in Z^{n}(L(\mathcal{X}, I))$ and $\varphi(z)=v$.

One can see that $i_{B}\left(B^{n}(L(\mathcal{X}, I))\right) \subseteq \operatorname{Ker} \varphi$, since $\widetilde{h\left(d_{n-1}\right)}$ is surjective and $\left[h\left(j_{n}\right) \circ h\left(d_{n-1}\right)\right](y)=0$ for any $y \in L\left(X_{n-1}, I\right)$. Suppose $z \in \operatorname{Ker} \varphi$, hence $\left[h\left(j_{n}\right) \circ i_{Z}\right](z)=0$. This implies that $i_{Z}(z) \in \operatorname{Ker} h\left(j_{n}\right)=\operatorname{Im} h\left(d_{n-1}\right)$, so that there is $y \in L\left(X_{n-1}, I\right)$ such that $h\left(d_{n-1}\right)(y)=i_{Z}(z)$. Since $i_{Z}$ is injective, $z=i_{B}\left(\widetilde{\left(d_{n-1}\right)}(y)\right)$. Thus $\operatorname{Ker} \varphi=i_{B}\left[B^{n}(L(\mathcal{X}, I))\right]$. The proof is complete.

Note that this is a strengthening of [8, Lemma 1.1 and Corollary 1.3] where $I=\mathbb{C}$. Let us state precisely the general result for $I=\mathbb{C}$.

Corollary 4.9. Let $\mathcal{X}$ be a chain complex of Fréchet (resp. Banach) spaces and $\mathcal{X}^{\prime}$ the strong dual cochain complex. Then the following are equivalent:

(1) $H_{n}(\mathcal{X})=\operatorname{Ker} d_{n-1} / \operatorname{Im} d_{n}$ is a Fréchet (resp. Banach) space;

(2) $B_{n}(\mathcal{X})=\operatorname{Im} d_{n}$ is closed in $X_{n}$;

(3) $d_{n}$ has closed range;

(4) the dual map $d^{n}=d_{n}^{*}$ has closed range;

(5) $B^{n+1}\left(\mathcal{X}^{\prime}\right)=\operatorname{Im} d_{n}^{*}$ is strongly closed in $\left(X_{n+1}\right)^{\prime}$.

In the category of Banach spaces, they are equivalent to:

(6) $B^{n+1}\left(\mathcal{X}^{\prime}\right)$ is a Banach space;

(7) $H^{n+1}\left(\mathcal{X}^{\prime}\right)=\operatorname{Ker} d_{n+1}^{*} / \operatorname{Im} d_{n}^{*}$ is a Banach space.

Moreover, whenever $H_{n}(\mathcal{X})$ and $H^{n}\left(\mathcal{X}^{\prime}\right)$ are Banach spaces, then

$$
H^{n}\left(\mathcal{X}^{\prime}\right) \cong H_{n}(\mathcal{X})^{\prime} .
$$

Proof. We can consider the chain complex

$$
\cdots \leftarrow 0 \longleftarrow X_{n-1} \stackrel{d_{n-1}}{\longleftarrow} X_{n} \stackrel{d_{n}}{\longleftarrow} X_{n+1} \leftarrow 0 \leftarrow \cdots
$$

where all but the three $\mathcal{X}$ terms are replaced by zero. The equivalence of (1)-(4) is a part of [4, Theorem 8.6.13], and (5) is clearly equivalent to (4). Statements (6) and (7) are also clear. 
For the last statement, note that $d_{n-1}$ has closed range because $H^{n}\left(\mathcal{X}^{\prime}\right)=$ $\operatorname{Ker} d_{n}^{*} / \operatorname{Im} d_{n-1}^{*}$ being a Banach space implies $d_{n-1}^{*}$ has closed range, while $d_{n}$ having closed range follows from $H_{n}(\mathcal{X})=\operatorname{Ker} d_{n-1} / \operatorname{Im} d_{n}$ being a Banach space. Therefore we can apply Theorem 4.8 to this complex with $I=\mathbb{C}$ to get $H^{n}\left(\mathcal{X}^{\prime}\right) \cong H_{n}(\mathcal{X})^{\prime}$.

Note that the previous result implies in particular that for a chain complex $\mathcal{X}$ of Banach spaces, the $H_{n}(\mathcal{X})$ are Banach spaces for all $n$ if and only if the $H^{n}\left(\mathcal{X}^{\prime}\right)$ are Banach spaces for all $n$.

5. The Künneth formula. The proof of the Künneth formula we will give is an adaptation of the proof given in [13] to the topological case. A part of the topological requirements will be verified using the following result, which is very close to [1, Lemma 7.1.32] or [7, Lemma 0.5.9]. We recall that a map $T$ between topological spaces is relatively open if it is open when seen as mapping into its image.

Lemma 5.1. Let $\mathcal{X}$ and $\mathcal{Y}$ be chain complexes in $\mathcal{F} r($ resp. in $\mathcal{B}$ an $)$ and let $\varphi: \mathcal{X} \rightarrow \mathcal{Y}$ be a continuous morphism of complexes of Fréchet (resp. Banach) spaces. Suppose that for some $n$,

$$
\varphi_{*}=H_{n}(\varphi): H_{n}(\mathcal{X}) \rightarrow H_{n}(\mathcal{Y})
$$

has closed range. Then $\varphi_{*}$ is relatively open. In particular, if $\varphi_{*}$ is surjective, then it is open.

Proof. Let $W=\varphi_{*}\left(H_{n}(\mathcal{X})\right)$ and let $\sigma_{\mathcal{Y}}: Z_{n}(\mathcal{Y}) \rightarrow H_{n}(\mathcal{Y})$ be the quotient map. As $W$ is a closed subspace of $H_{n}(\mathcal{Y}),(\sigma \mathcal{Y})^{-1}(W)=V$ is a closed subspace of $Z_{n}(\mathcal{Y})$ which contains $B_{n}(\mathcal{Y})=(\sigma \mathcal{Y})^{-1}(0)$. Denote by $d$ the boundary maps on $\mathcal{X}$ and by $d_{\mathcal{Y}}$ the boundary maps on $\mathcal{Y}$, and consider the map

$$
\psi: \operatorname{Ker} d_{n-1} \oplus Y_{n+1} \rightarrow V, \quad(x, y) \mapsto \varphi_{n}(x)+\left(d_{\mathcal{Y}}\right)_{n}(y) .
$$

By assumption $\varphi_{*}$ maps $H_{n}(\mathcal{X})$ onto $W$, which implies that $\psi$ is a surjective morphism of Fréchet spaces. Therefore, by the Open Mapping Theorem, $\psi$ is open.

Let $\widetilde{\varphi}_{*}: H_{n}(\mathcal{X}) \rightarrow W$ be $\varphi$ seen as mapping into its range. Consider the diagram

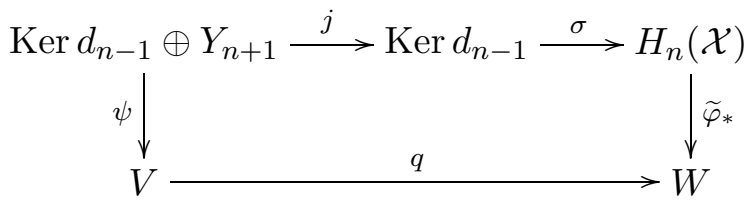

in which $j$ is a projection onto a direct summand, $\sigma$ is the natural projection and $q: V \rightarrow W$ is defined by $q(v)=\sigma_{\mathcal{Y}}(v)$ in $W$. Obviously this diagram is commutative. Note that $q$ is itself a projection as $B_{n}(\mathcal{X}) \subset V$, and therefore 
$q$ is an open map. As $\psi$ is also an open map, so is $q \circ \psi=\widetilde{\varphi}_{*} \circ \sigma \circ j$. Since $\sigma \circ j$ is continuous, $\widetilde{\varphi}_{*}$ is open, which is the same as $\varphi_{*}$ being relatively open.

Theorem 5.2. Let $\mathcal{X}$ and $\mathcal{Y}$ be bounded chain complexes in $\mathcal{F r}$ (resp. in $\mathcal{B}$ an) such that all boundary maps have closed range. Suppose that the following exact sequences of Fréchet (resp. Banach) spaces are topologically pure for all $n$ :

$$
\begin{aligned}
& 0 \rightarrow Z_{n}(\mathcal{X}) \stackrel{i}{\rightarrow} X_{n} \stackrel{\widetilde{d}_{\mathcal{X}}}{\rightarrow} B_{n-1}(\mathcal{X}) \rightarrow 0 \\
& 0 \rightarrow B_{n}(\mathcal{X}) \stackrel{j}{\rightarrow} Z_{n}(\mathcal{X}) \stackrel{\sigma}{\rightarrow} H_{n}(\mathcal{X}) \rightarrow 0
\end{aligned}
$$

Suppose also that one of the following two cases is satisfied.

CASE 1. The following exact sequences of Fréchet (resp. Banach) spaces are topologically pure for all $n$ :

$$
\begin{aligned}
& 0 \rightarrow Z_{n}(\mathcal{Y}) \stackrel{i}{\rightarrow} Y_{n} \stackrel{\widetilde{d}_{\mathcal{Y}}}{\rightarrow} B_{n-1}(\mathcal{Y}) \rightarrow 0, \\
& 0 \rightarrow B_{n}(\mathcal{Y}) \stackrel{j}{\rightarrow} Z_{n}(\mathcal{Y}) \stackrel{\sigma}{\rightarrow} H_{n}(\mathcal{Y}) \rightarrow 0 .
\end{aligned}
$$

CASE $2 . Z_{n}(\mathcal{X})$ and $B_{n}(\mathcal{X})$ are strictly flat for all $n$.

Then, up to topological isomorphism,

$$
\bigoplus_{m+q=n}\left[H_{m}(\mathcal{X}) \widehat{\otimes} H_{q}(\mathcal{Y})\right]=H_{n}(\mathcal{X} \widehat{\otimes} \mathcal{Y})
$$

Proof. We regard the families $Z_{n}(\mathcal{X})$ and $D_{n}=B_{n-1}(\mathcal{X})$ as bounded chain complexes of Fréchet (resp. Banach) spaces with zero boundary. Since, for all $n,(10)$ is topologically pure, the sequence of chain complexes

$$
0 \rightarrow \mathcal{Z}(\mathcal{X}) \stackrel{i}{\rightarrow} \mathcal{X} \stackrel{\widetilde{d}_{\mathcal{X}}}{\rightarrow} \mathcal{D} \rightarrow 0
$$

is also topologically pure, and, for all $m$ and $q$,

$$
0 \rightarrow\left(Z_{m}(\mathcal{X}) \widehat{\otimes} Y_{q}\right) \stackrel{i \otimes 1_{Y_{q}}}{\longrightarrow}\left(X_{m} \widehat{\otimes} Y_{q}\right) \stackrel{\widetilde{d}_{\mathcal{X}} \otimes 1_{Y_{q}}}{\longrightarrow}\left(D_{m} \widehat{\otimes} Y_{q}\right) \rightarrow 0
$$

is exact. Note that

$$
(\mathcal{X} \widehat{\otimes} \mathcal{Y})_{n}=\bigoplus_{m+q=n} X_{m} \widehat{\otimes} Y_{q}
$$

Therefore the sequence

$$
0 \rightarrow \mathcal{Z}(\mathcal{X}) \widehat{\otimes} \mathcal{Y} \stackrel{i \otimes 1 \mathcal{Y}}{\longrightarrow} \mathcal{X} \widehat{\otimes} \mathcal{Y} \stackrel{\widetilde{d}_{\mathcal{X}} \otimes 1 \mathcal{Y}}{\longrightarrow} \mathcal{D} \widehat{\otimes} \mathcal{Y} \rightarrow 0
$$

is also an exact sequence of chain complexes of Fréchet (resp. Banach) spaces. This leads to a long exact homology sequence

$$
\begin{array}{r}
\cdots \rightarrow H_{n+1}(\mathcal{D} \widehat{\otimes} \mathcal{Y}) \stackrel{E_{n+1}}{\longrightarrow} H_{n}(\mathcal{Z}(\mathcal{X}) \widehat{\otimes} \mathcal{Y}) \stackrel{H_{n}(i \otimes 1 \mathcal{Y})}{\longrightarrow} H_{n}(\mathcal{X} \widehat{\otimes} \mathcal{Y}) \\
\stackrel{H_{n}\left(\widetilde{d}_{\mathcal{X}} \otimes 1 \mathcal{Y}\right)}{\longrightarrow} H_{n}(\mathcal{D} \widehat{\otimes} \mathcal{Y}) \stackrel{E_{n}}{\longrightarrow} H_{n-1}(\mathcal{Z}(\mathcal{X}) \widehat{\otimes} \mathcal{Y}) \rightarrow \cdots
\end{array}
$$


It follows from [7, Theorem 0.5.7] that the induced maps $E_{n}, H_{n}(i \otimes 1 \mathcal{Y})$ and $H_{n}\left(\widetilde{d}_{\mathcal{X}} \otimes 1 \mathcal{Y}\right)$ in this long exact sequence are continuous.

By assumption (11), for all $m$, the sequence

$$
0 \rightarrow D_{m+1} \stackrel{j}{\rightarrow} Z_{m}(\mathcal{X}) \stackrel{\sigma}{\rightarrow} H_{m}(\mathcal{X}) \rightarrow 0
$$

is topologically pure. Therefore, since $H_{q}(\mathcal{Y})$ is a Fréchet (resp. Banach) space, the sequence

$$
0 \rightarrow D_{m+1} \widehat{\otimes} H_{q}(\mathcal{Y}) \stackrel{j \otimes 1_{H_{q}(\mathcal{Y})}}{\longrightarrow} Z_{m}(\mathcal{X}) \widehat{\otimes} H_{q}(\mathcal{Y}) \stackrel{\sigma \otimes 1_{H_{q}(\mathcal{Y})}}{\longrightarrow} H_{m}(\mathcal{X}) \widehat{\otimes} H_{q}(\mathcal{Y}) \rightarrow 0
$$

is exact. Now take the direct sum over $m+q=n$ to obtain

$$
\begin{aligned}
& 0 \rightarrow \bigoplus_{m+q=n}\left[D_{m+1} \widehat{\otimes} H_{q}(\mathcal{Y})\right] \\
& \quad \stackrel{j \otimes 1_{H}}{\longrightarrow} \bigoplus_{m+q=n}\left[Z_{m}(\mathcal{X}) \widehat{\otimes} H_{q}(\mathcal{Y})\right] \stackrel{\sigma \otimes 1_{H}}{\longrightarrow} \bigoplus_{m+q=n}\left[H_{m}(\mathcal{X}) \widehat{\otimes} H_{q}(\mathcal{Y})\right] \rightarrow 0
\end{aligned}
$$

The vertical maps $p_{1}$ and $p_{2}$ of the following diagram are the topological isomorphisms arising from Proposition 3.3 or Proposition 4.5:

$$
\begin{aligned}
& 0 \rightarrow \bigoplus_{m+q=n}\left[D_{m+1} \widehat{\otimes} H_{q}(\mathcal{Y})\right] \stackrel{j \otimes 1_{H}}{\longrightarrow} \bigoplus_{m+q=n}\left[Z_{m}(\mathcal{X}) \widehat{\otimes} H_{q}(\mathcal{Y})\right] \stackrel{\sigma \otimes 1_{H}}{\longrightarrow} \bigoplus_{m+q=n}\left[H_{m}(\mathcal{X}) \widehat{\otimes} H_{q}(\mathcal{Y})\right] \rightarrow 0 \\
& p_{1} \mid \downarrow{ }^{p_{2}} \\
& H_{n+1}(\mathcal{D} \widehat{\otimes} \mathcal{Y}) \stackrel{E_{n+1}}{\longrightarrow} H_{n}(\mathcal{Z}(K) \widehat{\otimes} \mathcal{Y})
\end{aligned}
$$

Note that all spaces of the diagram are Fréchet (resp. Banach) spaces.

Now we have to prove that the square is commutative. Since all linear operators are continuous, it is enough to show that for a tensor $\sum x_{i} \otimes q_{\mathcal{Y}}\left(y_{i}\right)$ $\in D_{m+1} \widehat{\otimes} H_{q}(\mathcal{Y})$, where $q_{\mathcal{Y}}\left(y_{i}\right)$ is the homology class of $y_{i}$,

$$
E_{n+1} \circ p_{1}\left(\sum x_{i} \otimes q \mathcal{Y}\left(y_{i}\right)\right)=p_{2} \circ\left(j \otimes 1_{H}\right)\left(\sum x_{i} \otimes q \mathcal{Y}\left(y_{i}\right)\right) .
$$

On the right hand side, we have

$$
p_{2} \circ\left(j \otimes 1_{H}\right)\left(\sum x_{i} \otimes q_{\mathcal{Y}}\left(y_{i}\right)\right)=q_{\mathcal{Z}(K) \widehat{\otimes} \mathcal{Y}}\left(\sum x_{i} \otimes y_{i}\right)
$$

(using Proposition 3.3 or 4.5 for the definition of $p_{2}$ ).

Similarly, on the left hand side, we have

$$
p_{1}\left(\sum x_{i} \otimes q_{\mathcal{Y}}\left(y_{i}\right)\right)=q_{\mathcal{D} \widehat{\otimes} \mathcal{Y}}\left(\sum x_{i} \otimes y_{i}\right) .
$$

The connecting homomorphism $E_{n+1}$ from the short exact sequence (14) is defined as follows on the homology class $q_{\mathcal{D} \widehat{\otimes} \mathcal{Y}}\left(\sum x_{i} \otimes y_{i}\right)$ : pull the cycle

$$
\sum x_{i} \otimes y_{i} \in D_{m+1} \widehat{\otimes} Y_{q}
$$

back to a chain

$$
\sum u_{i} \otimes y_{i} \in X_{m+1} \widehat{\otimes} Y_{q}
$$


where $d \mathcal{X}\left(u_{i}\right)=x_{i}$; take its boundary

$$
\sum d_{\mathcal{X} \widehat{\otimes} \mathcal{Y}}\left(u_{i} \otimes y_{i}\right)=\sum d_{\mathcal{X}}\left(u_{i}\right) \otimes y_{i}=\sum x_{i} \otimes y_{i} \in X_{m} \widehat{\otimes} Y_{q}
$$

pull this boundary to $Z_{m}(\mathcal{X}) \widehat{\otimes} Y_{q}$ and take the homology class of the result. This gives the homology class of $\sum x_{i} \otimes y_{i}$ in $H_{n}(\mathcal{Z}(\mathcal{X}) \widehat{\otimes} \mathcal{Y})$. Therefore the square commutes.

Hence $\operatorname{Ker} E_{n}=0$ and, up to topological isomorphism,

$$
\text { Coker } E_{n+1}=\bigoplus_{m+q=n}\left[H_{m}(\mathcal{X}) \widehat{\otimes} H_{q}(\mathcal{Y})\right] \text {. }
$$

Thus the following two facts: $\operatorname{Ker} E_{n}=0$ for all $n$ and the exactness of the sequence (15), imply the exactness of short exact sequences

$$
0 \rightarrow H_{n+1}(\mathcal{D} \widehat{\otimes} \mathcal{Y}) \stackrel{E_{n+1}}{\longrightarrow} H_{n}(\mathcal{Z}(\mathcal{X}) \widehat{\otimes} \mathcal{Y}) \stackrel{H_{n}(i \otimes 1 \mathcal{Y})}{\longrightarrow} H_{n}(\mathcal{X} \widehat{\otimes} \mathcal{Y}) \rightarrow 0
$$

for all $n$. Hence, by Lemma 5.1, for the continuous morphism of complexes of Fréchet (resp. Banach) spaces

$$
\varphi=i \otimes 1_{\mathcal{Y}}: \mathcal{Z}(\mathcal{X}) \widehat{\otimes} \mathcal{Y} \rightarrow \mathcal{X} \widehat{\otimes} \mathcal{Y},
$$

the surjective maps $H_{n}(i \otimes 1 \mathcal{Y})$ are open for all $n$. Therefore the induced map

$$
\overline{H_{n}(i \otimes 1 \mathcal{Y})}: H_{n}(\mathcal{Z}(\mathcal{X}) \widehat{\otimes} \mathcal{Y}) / \operatorname{Ker} H_{n}(i \otimes 1 \mathcal{Y}) \rightarrow H_{n}(\mathcal{X} \widehat{\otimes} \mathcal{Y})
$$

is a topological isomorphism. Hence, up to topological isomorphism,

$$
\begin{aligned}
\bigoplus_{m+q=n}\left[H_{m}(\mathcal{X}) \widehat{\otimes} H_{q}(\mathcal{Y})\right] & =\text { Coker } E_{n+1}=H_{n}(\mathcal{Z}(\mathcal{X}) \widehat{\otimes} \mathcal{Y}) / \operatorname{Im} E_{n+1} \\
& =H_{n}(\mathcal{Z}(\mathcal{X}) \widehat{\otimes} \mathcal{Y}) / \operatorname{Ker} H_{n}(i \otimes 1 \mathcal{Y})=H_{n}(\mathcal{X} \widehat{\otimes} \mathcal{Y}) .
\end{aligned}
$$

Corollary 5.3. Let $\mathcal{X}$ and $\mathcal{Y}$ be bounded chain complexes of nuclear Fréchet spaces and continuous operators such that all boundary maps have closed range. Then, up to topological isomorphism,

$$
\bigoplus_{m+q=n}\left[H_{m}(\mathcal{X}) \widehat{\otimes} H_{q}(\mathcal{Y})\right]=H_{n}(\mathcal{X} \widehat{\otimes} \mathcal{Y})
$$

Proof. By Theorems A.1.6 and A.1.5 of [5], for all $n$, the short exact sequences of nuclear Fréchet spaces (10)-(13) are topologically pure in $\mathcal{F} r$. The result follows from Theorem 5.2 (Case 1).

Corollary 5.4. Let $\mathcal{X}$ and $\mathcal{Y}$ be bounded chain complexes in $\mathcal{B}$ an such that all boundary maps have closed range. Suppose that, for all $n, B_{n}(\mathcal{X})$ and $H_{n}(\mathcal{X})$ are strictly flat. Then, up to topological isomorphism,

$$
\bigoplus_{m+q=n}\left[H_{m}(\mathcal{X}) \widehat{\otimes} H_{q}(\mathcal{Y})\right]=H_{n}(\mathcal{X} \widehat{\otimes} \mathcal{Y})
$$


Proof. By [7, Proposition VII.1.17], $B_{n}(\mathcal{X})$ and $H_{n}(\mathcal{X})$ strictly flat implies that $Z_{n}(\mathcal{X})$ is strictly flat as well. By Lemma 4.3 , strict flatness of $B_{n-1}(\mathcal{X})$ and $H_{n}(\mathcal{X})$ implies that the short exact sequences of Banach spaces (10), (11) are topologically pure in $\mathcal{B}$ an . The result follows from Theorem 5.2 (Case 2).

6. External products of Hochschild homology. Let $\mathcal{A}$ be a Fréchet (resp. Banach) algebra and $X$ be a Fréchet (resp. Banach) $\mathcal{A}$-bimodule. Let us recall the definition of the standard homological chain complex $\mathcal{C}_{\sim}(\mathcal{A}, X)$. For $n \geq 0$, let $C_{n}(\mathcal{A}, X)$ denote the projective tensor product $X \widehat{\otimes} \mathcal{A}^{\widehat{\otimes}^{n}}$. The elements of $C_{n}(\mathcal{A}, X)$ are called $n$-chains. Let the differential $d_{n}: C_{n+1} \rightarrow$ $C_{n}$ be given by

$$
\begin{aligned}
d_{n}\left(x \otimes a_{1} \otimes \cdots \otimes a_{n+1}\right)= & x \cdot a_{1} \otimes \cdots \otimes a_{n+1} \\
& +\sum_{k=1}^{n}(-1)^{k}\left(x \otimes a_{1} \otimes \cdots \otimes a_{k} a_{k+1} \otimes \cdots \otimes a_{n+1}\right) \\
& +(-1)^{n+1}\left(a_{n+1} \cdot x \otimes a_{1} \otimes \cdots \otimes a_{n}\right)
\end{aligned}
$$

with $d_{-1}$ the null map. The space of boundaries $B_{n}\left(\mathcal{C}_{\sim}(\mathcal{A}, X)\right)=\operatorname{Im} d_{n}$ is denoted by $B_{n}(\mathcal{A}, X)$ and the space of cycles $Z_{n}\left(\mathcal{C}_{\sim}(\mathcal{A}, X)\right)=\operatorname{Ker} d_{n-1}$ is denoted by $Z_{n}(\mathcal{A}, X)$. The homology groups of this complex $H_{n}\left(\mathcal{C}_{\sim}(\mathcal{A}, X)\right)=$ $Z_{n}(\mathcal{A}, X) / B_{n}(\mathcal{A}, X)$ are called the Hochschild homology groups of $\mathcal{A}$ with coefficients in $X$ and denoted by $\mathcal{H}_{n}(\mathcal{A}, X)$ [7, Definition II.5.28].

The Hochschild cohomology groups $\mathcal{H}^{n}\left(\mathcal{A}, X^{\prime}\right)$ of the Banach algebra $\mathcal{A}$ with coefficients in the dual $\mathcal{A}$-bimodule $X^{\prime}$ are topologically isomorphic to the cohomology groups $H^{n}\left(\left(\mathcal{C}_{\sim}(\mathcal{A}, X)\right)^{\prime}\right)$ of the dual complex $\left(\mathcal{C}_{\sim}(\mathcal{A}, X)\right)^{\prime}$ [7, Definition I.3.2 and Proposition II.5.27].

Let $\mathcal{A}$ be a unital Fréchet algebra. We put $\beta_{n}(\mathcal{A})=\mathcal{A}^{\widehat{\otimes}^{n+2}}, n \geq 0$, and let $d_{n}: \beta_{n+1}(\mathcal{A}) \rightarrow \beta_{n}(\mathcal{A})$ be given by

$$
d_{n}\left(a_{0} \otimes \cdots \otimes a_{n+2}\right)=\sum_{k=0}^{n+1}(-1)^{k}\left(a_{0} \otimes \cdots \otimes a_{k} a_{k+1} \otimes \cdots \otimes a_{n+2}\right) .
$$

By [7, Proposition III.2.9], the complex over $\mathcal{A}, \pi: \beta(\mathcal{A}) \rightarrow \mathcal{A}: a \otimes b \mapsto a b$, where $\beta(\mathcal{A})$ denotes

$$
0 \leftarrow \beta_{0}(\mathcal{A}) \stackrel{d_{0}}{\longleftarrow} \beta_{1}(\mathcal{A}) \stackrel{d_{1}}{\longleftarrow} \cdots \leftarrow \beta_{n}(\mathcal{A}) \stackrel{d_{n}}{\longleftarrow} \beta_{n+1}(\mathcal{A}) \leftarrow \ldots,
$$

is a projective resolution of the $\mathcal{A}$-bimodule $\mathcal{A}$. $\beta(\mathcal{A})$ is called the bar resolution of $\mathcal{A}$. The complex has a contracting homotopy $s_{n}: \beta_{n}(\mathcal{A}) \rightarrow \beta_{n+1}(\mathcal{A})$ $(n \geq 1)$, given by

$$
s_{n}\left(a_{0} \otimes a_{1} \otimes \cdots \otimes a_{n+1}\right)=1 \otimes a_{0} \otimes a_{1} \otimes \cdots \otimes a_{n+1},
$$

which is to say that $d_{n} s_{n}+s_{n-1} d_{n-1}=1_{\beta_{n}(\mathcal{A})}$. 
Proposition 6.1. Let $A_{1}$ and $A_{2}$ be unital Fréchet algebras, let $0 \leftarrow$ $X \stackrel{\varepsilon_{1}}{\longleftarrow} \mathcal{X}$ be a projective resolution of $X \in A_{1}$-mod and $0 \leftarrow Y \stackrel{\varepsilon_{2}}{\longleftarrow} \mathcal{Y}$ be a projective resolution of $Y \in A_{2}$-mod. Then $0 \leftarrow X \widehat{\otimes} Y \stackrel{\varepsilon_{1} \otimes \varepsilon_{2}}{\longleftarrow} \mathcal{X} \widehat{\otimes} \mathcal{Y}$ is a projective resolution of $X \widehat{\otimes} Y \in A_{1} \widehat{\otimes} A_{2}$-mod.

Proof. The proof requires only minor modifications of that of [13, Proposition X.7.1].

Note that the statement of Proposition 6.1 is also true in the category of bimodules.

Theorem 6.2. Let $\mathcal{A}$ and $\mathcal{B}$ be unital Fréchet algebras, let $X$ be a Fréchet $\mathcal{A}$-bimodule and let $Y$ be a Fréchet $\mathcal{B}$-bimodule. Then, up to topological isomorphism,

$$
\mathcal{H}_{n}(\mathcal{A} \widehat{\otimes} \mathcal{B}, X \widehat{\otimes} Y)=H_{n}\left(\mathcal{C}_{\sim}(\mathcal{A}, X) \widehat{\otimes} \mathcal{C}_{\sim}(\mathcal{B}, Y)\right) .
$$

Proof. Let $\beta(\mathcal{A})$ and $\beta(\mathcal{B})$ be the bar resolutions of $\mathcal{A}$ and $\mathcal{B}$. Since the bar resolution $\beta(\mathcal{A})$ is an $\mathcal{A}$-biprojective resolution of $\mathcal{A}$ and $\beta(\mathcal{B})$ is a $\mathcal{B}$-biprojective resolution of $\mathcal{B}$, by Proposition 6.1 their projective tensor product $\beta(\mathcal{A}) \widehat{\otimes} \beta(\mathcal{B})$ is an $\mathcal{A} \widehat{\otimes} \mathcal{B}$-biprojective resolution of $\mathcal{A} \widehat{\otimes} \mathcal{B}$.

For a unital Fréchet algebra $\mathcal{U}$ and for a Fréchet $\mathcal{U}$-bimodule $Z$, recall [7, Theorem III.4.25] that the Hochschild chain complex $\mathcal{C}_{\sim}(\mathcal{U}, Z)$ is isomorphic to $Z \widehat{\otimes}_{\mathcal{U}}$ e $\beta(\mathcal{U})$ and

$$
\mathcal{H}_{n}(\mathcal{U}, Z)=\operatorname{Tor}_{n}^{\mathcal{U}}(Z, \mathcal{U})=H_{n}\left(Z \widehat{\otimes}_{\mathcal{U}} \beta(\mathcal{U})\right) .
$$

Therefore, since the $n$th derived functor $\operatorname{Tor}_{n}^{\mathcal{U}}(\cdot, \mathcal{U})$ does not depend on the choice of a $\mathcal{U}$-biprojective resolution of $\mathcal{U}$, up to topological isomorphism,

$$
\begin{aligned}
\mathcal{H}_{n}(\mathcal{A} \widehat{\otimes} \mathcal{B}, X \widehat{\otimes} Y) & =\operatorname{Tor}_{n}^{(\mathcal{A} \widehat{\otimes} \mathcal{B})^{\mathrm{e}}}(X \widehat{\otimes} Y, \mathcal{A} \widehat{\otimes} \mathcal{B}) \\
& =H_{n}\left((X \widehat{\otimes} Y) \widehat{\otimes}_{(\mathcal{A} \widehat{\otimes} \mathcal{B})^{\mathrm{e}}} \beta(\mathcal{A} \widehat{\otimes} \mathcal{B})\right) \\
& =H_{n}\left((X \widehat{\otimes} Y) \widehat{\otimes}_{(\mathcal{A} \widehat{\otimes} \mathcal{B})^{\mathrm{e}}}(\beta(\mathcal{A}) \widehat{\otimes} \beta(\mathcal{B}))\right) .
\end{aligned}
$$

By [7, Proposition II.3.13], one can see that the following chain complexes are isomorphic:

$$
\begin{aligned}
(X \widehat{\otimes} Y) \widehat{\otimes}_{(\mathcal{A} \widehat{\otimes} \mathcal{B})^{\mathrm{e}}}(\beta(\mathcal{A}) \widehat{\otimes} \beta(\mathcal{B})) & \cong\left(X \widehat{\otimes}_{\mathcal{A}^{\mathrm{e}}} \beta(\mathcal{A})\right) \widehat{\otimes}\left(Y \widehat{\otimes}_{\mathcal{B}^{\mathrm{e}}} \beta(\mathcal{B})\right) \\
& \cong \mathcal{C}_{\sim}(\mathcal{A}, X) \widehat{\otimes} \mathcal{C}_{\sim}(\mathcal{B}, Y) .
\end{aligned}
$$

Thus, up to topological isomorphism,

$$
\begin{aligned}
\mathcal{H}_{n}(\mathcal{A} \widehat{\otimes} \mathcal{B}, X \widehat{\otimes} Y) & =H_{n}\left((X \widehat{\otimes} Y) \widehat{\otimes}_{(\mathcal{A} \widehat{\otimes} \mathcal{B})^{\mathrm{e}}}(\beta(\mathcal{A}) \widehat{\otimes} \beta(\mathcal{B}))\right) \\
& =H_{n}\left(\mathcal{C}_{\sim}(\mathcal{A}, X) \widehat{\otimes} \mathcal{C}_{\sim}(\mathcal{B}, Y)\right) .
\end{aligned}
$$

Corollary 6.3. Let $\mathcal{A}$ and $\mathcal{B}$ be unital nuclear Fréchet algebras, let $X$ be a nuclear Fréchet $\mathcal{A}$-bimodule and let $Y$ be a nuclear Fréchet 
$\mathcal{B}$-bimodule. Suppose that all boundary maps of the standard homology complexes $\mathcal{C}_{\sim}(\mathcal{A}, X)$ and $\mathcal{C}_{\sim}(\mathcal{B}, Y)$ have closed range. Then, up to topological isomorphism,

$$
\mathcal{H}_{n}(\mathcal{A} \widehat{\otimes} \mathcal{B}, X \widehat{\otimes} Y)=\bigoplus_{m+q=n}\left[\mathcal{H}_{m}(\mathcal{A}, X) \widehat{\otimes} \mathcal{H}_{q}(\mathcal{B}, Y)\right] .
$$

Proof. By [16, Proposition III.50.1], the projective tensor product of nuclear Fréchet spaces is a nuclear Fréchet space. The result follows from Theorem 6.2 and Corollary 5.3.

Corollary 6.4. Let $\mathcal{A}$ and $\mathcal{B}$ be unital Banach algebras, let $X$ be a Banach $\mathcal{A}$-bimodule and let $Y$ be a Banach $\mathcal{B}$-bimodule. Suppose that all boundary maps of the standard homology complexes $\mathcal{C}_{\sim}(\mathcal{A}, X)$ and $\mathcal{C}_{\sim}(\mathcal{B}, Y)$ have closed range and, for all $n, B_{n}(\mathcal{A}, X)$ and $\mathcal{H}_{n}(\mathcal{A}, X)$ are strictly flat. Then, up to topological isomorphism,

$$
\begin{aligned}
\mathcal{H}_{n}(\mathcal{A} \widehat{\otimes} \mathcal{B}, X \widehat{\otimes} Y) & =\bigoplus_{m+q=n}\left[\mathcal{H}_{m}(\mathcal{A}, X) \widehat{\otimes} \mathcal{H}_{q}(\mathcal{B}, Y)\right], \\
\mathcal{H}^{n}\left(\mathcal{A} \widehat{\otimes} \mathcal{B},(X \widehat{\otimes} Y)^{\prime}\right) & =\bigoplus_{m+q=n}\left[\mathcal{H}_{m}(\mathcal{A}, X) \widehat{\otimes} \mathcal{H}_{q}(\mathcal{B}, Y)\right]^{\prime} .
\end{aligned}
$$

Proof. The first isomorphism follows from Theorem 6.2 and Corollary 5.4. By [7, Proposition II.5.27],

$$
\mathcal{H}^{n}\left(\mathcal{A} \widehat{\otimes} \mathcal{B},(X \widehat{\otimes} Y)^{\prime}\right) \cong H^{n}\left(\left(\mathcal{C}_{\sim}(\mathcal{A} \widehat{\otimes} \mathcal{B}, X \widehat{\otimes} Y)\right)^{\prime}\right) .
$$

By Corollary 4.9, since the $\mathcal{H}_{n}(\mathcal{A} \widehat{\otimes} \mathcal{B}, X \widehat{\otimes} Y)$ are Banach spaces, $H^{n}\left(\left(\mathcal{C}_{\sim}(\mathcal{A} \widehat{\otimes} \mathcal{B}, X \widehat{\otimes} Y)\right)^{\prime}\right) \cong\left(H_{n}\left(\mathcal{C}_{\sim}(\mathcal{A} \widehat{\otimes} \mathcal{B}, X \widehat{\otimes} Y)\right)\right)^{\prime} \cong\left(\mathcal{H}_{n}(\mathcal{A} \widehat{\otimes} \mathcal{B}, X \widehat{\otimes} Y)\right)^{\prime}$. Therefore

$$
\mathcal{H}^{n}\left(\mathcal{A} \widehat{\otimes} \mathcal{B},(X \widehat{\otimes} Y)^{\prime}\right) \cong\left(\mathcal{H}_{n}(\mathcal{A} \widehat{\otimes} \mathcal{B}, X \widehat{\otimes} Y)\right)^{\prime}
$$

7. Some strictly projective Banach spaces and split short exact sequences. From now on, let $\mathcal{A}=\ell^{1}\left(\mathbb{Z}_{+}\right)$, where

$$
\ell^{1}\left(\mathbb{Z}_{+}\right)=\left\{\left(a_{n}\right)_{n=0}^{\infty}: \sum_{n=0}^{\infty}\left|a_{n}\right|<\infty\right\}
$$

is the unital semigroup Banach algebra of $\mathbb{Z}_{+}$with convolution multiplication and norm $\left\|\left(a_{n}\right)_{n=0}^{\infty}\right\|=\sum_{n=0}^{\infty}\left|a_{n}\right|$. Recall that $\ell^{1}\left(\mathbb{Z}_{+}\right)$is isometrically isomorphic to the unital commutative Banach algebra

$$
A^{+}(\overline{\mathbb{D}})=\left\{f=\sum_{n=0}^{\infty} a_{n} z^{n}: \sum_{n=0}^{\infty}\left|a_{n}\right|<\infty\right\}
$$

of absolutely convergent Taylor series on $\overline{\mathbb{D}}$ with pointwise multiplication and norm $\|f\|=\sum_{n=0}^{\infty}\left|a_{n}\right|$, where $\overline{\mathbb{D}}=\{z \in \mathbb{C}:|z| \leq 1\}$ is the closed disc. 
Let $\mathcal{I}=\ell^{1}(\mathbb{N})$ be the closed ideal of $\ell^{1}\left(\mathbb{Z}_{+}\right)$consisting of those elements with $a_{0}=0$.

We recall the definition of a strictly projective Banach space. Note that this is a special case of the notion of a strictly projective Banach module, where the Banach algebra is taken to be the complex numbers $\mathbb{C}$.

Definition 7.1. A Banach space $P$ is strictly projective if, for every pair of Banach spaces $E$ and $F$, for every surjective continuous linear operator $q: E \rightarrow F$ and for every continuous linear operator $\theta: P \rightarrow F$, there is a continuous linear operator $\vartheta: P \rightarrow E$ such that $q \circ \vartheta=\theta$.

In particular, recall that any short exact sequence of Banach spaces $0 \rightarrow X \rightarrow Y \rightarrow P \rightarrow 0$ with $P$ strictly projective splits.

Lemma 7.2. A Banach space is strictly projective if and only if it is a direct summand (i.e. a complemented subspace) of $\ell^{1}(S)$ for some set $S$.

Proof. This follows from [10, Proposition 2.f.7] and [9], as indicated in the remarks after [10, Proposition 2.f.7]. Alternatively, see [14, Proposition 3.2.3].

Proposition 7.3. Let $\mathcal{A}=\ell^{1}\left(\mathbb{Z}_{+}\right)$. Then the simplicial homology groups $\mathcal{H}_{n}(\mathcal{A}, \mathcal{A})$ are given by

$$
\begin{aligned}
& \mathcal{H}_{0}(\mathcal{A}, \mathcal{A}) \cong \mathcal{A}=\ell^{1}\left(\mathbb{Z}_{+}\right), \quad \mathcal{H}_{1}(\mathcal{A}, \mathcal{A}) \cong \mathcal{I}=\ell^{1}(\mathbb{N}), \\
& \mathcal{H}_{n}(\mathcal{A}, \mathcal{A}) \cong 0 \quad \text { for } n \geq 2,
\end{aligned}
$$

where $\cong$ denotes isomorphism of Banach spaces.

Proof. Since $\mathcal{A}$ is commutative, $B_{0}(\mathcal{A}, \mathcal{A})=\operatorname{Im} d_{0}=\{0\}$. Thus $\mathcal{H}_{0}(\mathcal{A}, \mathcal{A})$ $=Z_{0}(\mathcal{A}, \mathcal{A})=\mathcal{A}=\ell^{1}\left(\mathbb{Z}_{+}\right)$. It is proved in [6] that the cohomology groups $\mathcal{H}^{n}(\mathcal{A}, \mathcal{A})$ are trivial for $n \geq 2$, hence, by [8, Corollary 1.3], $\mathcal{H}_{n}(\mathcal{A}, \mathcal{A})=0$ for $n \geq 2$ and $B_{1}(\mathcal{A}, \mathcal{A})$ is closed. Thus $\mathcal{H}_{1}(\mathcal{A}, \mathcal{A})$ is a Banach space.

To determine $\mathcal{H}_{1}(\mathcal{A}, \mathcal{A})=C_{1}(\mathcal{A}, \mathcal{A}) / B_{1}(\mathcal{A}, \mathcal{A})$, firstly, note that since $\mathcal{A}$ is commutative, $Z_{1}(\mathcal{A}, \mathcal{A})=\operatorname{Ker} d_{0}=C_{1}(\mathcal{A}, \mathcal{A})=\mathcal{A} \widehat{\otimes} \mathcal{A}$. Thus, to prove that $\mathcal{H}_{1}(\mathcal{A}, \mathcal{A}) \cong \mathcal{I}=\ell^{1}(\mathbb{N})$, it is enough to show the exactness of the short sequence

$$
0 \rightarrow B_{1}(\mathcal{A}, \mathcal{A}) \stackrel{j}{\rightarrow} C_{1}(\mathcal{A}, \mathcal{A}) \stackrel{q}{\rightarrow} \mathcal{I} \rightarrow 0,
$$

where $j$ is the usual inclusion and the continuous linear operator

$$
q: \ell^{1}\left(\mathbb{Z}_{+}\right) \widehat{\otimes} \ell^{1}\left(\mathbb{Z}_{+}\right) \rightarrow \ell^{1}(\mathbb{N})
$$

is induced by

$$
q\left(z^{k} \otimes z^{l}\right)=\frac{l}{k+l} z^{k+l}
$$

when at least one of $k, l$ is not zero, and $q\left(z^{0} \otimes z^{0}\right)=0$. 
To prove our claim, first note that the boundary $d_{1}: \ell^{1}\left(\mathbb{Z}_{+}^{3}\right) \rightarrow \ell^{1}\left(\mathbb{Z}_{+}^{2}\right)$, given by

$$
d_{1}\left(z^{k} \otimes z^{l} \otimes z^{m}\right)=\left(z^{m+k} \otimes z^{l}\right)-\left(z^{k} \otimes z^{l+m}\right)+\left(z^{k+l} \otimes z^{m}\right),
$$

maps into the kernel of $q$ as

$q\left[d_{1}\left(z^{k} \otimes z^{l} \otimes z^{m}\right)\right]=\left[l z^{k+l+m}-(l+m) z^{k+l+m}+m z^{k+l+m}\right] /(k+l+m)=0$.

Here we have identified $C_{n}\left(\ell^{1}\left(\mathbb{Z}_{+}\right), \ell^{1}\left(\mathbb{Z}_{+}\right)\right)$with $\ell^{1}\left(\mathbb{Z}_{+}^{n+1}\right)$.

If we now take any $f$ in the kernel of $q$, then the following argument shows that $f$ is in the image of $d_{1}$. Clearly, $f$ is in $\operatorname{Ker} q$ if and only if its real and imaginary parts are. Therefore it is sufficient to prove that a real-valued $f$ in Ker $q$ is in the image of $d_{1}$. Clearly $f=\sum_{k=0}^{\infty} \sum_{j=0}^{\infty} a_{k, j}\left(z^{k} \otimes z^{j}\right) \in \operatorname{Ker} q$ if and only if for each $m \in \mathbb{Z}_{+}$, we have

$$
\sum_{j=0}^{m} j a_{m-j, j}=0 .
$$

Let $p_{m}(f)=\sum_{j=0}^{m}\left|a_{m-j, j}\right|$ so that $\|f\|=\sum_{m=0}^{\infty} p_{m}(f)$.

For each $m \in \mathbb{Z}^{+}$, we construct $b^{m}=\left(b_{i, j, k}^{m}\right) \in \ell^{1}\left(\mathbb{Z}_{+}^{3}\right)$, where $b_{i, j, k}^{m}=0$ if $i+j+k \neq m,\left(f-d_{1}\left(b^{m}\right)\right)_{m-j, j}=0$ for $0 \leq j \leq m$ and $\left\|b^{m}\right\| \leq p_{m}(f)$. Then $g=\sum_{m=0}^{\infty} b^{m}$ is a well defined element of $\ell^{1}\left(\mathbb{Z}_{+}^{3}\right)$ as $\sum_{m=0}^{\infty} p_{m}(f)=\|f\|$ and $d_{1}(g)=f$, proving that $f \in \operatorname{Im} d_{1}$, which completes the proof.

The construction proceeds as follows. Let $m$ be fixed.

STEP 1. If $\left\{j: a_{m-j, j} \neq 0\right\}$ is empty, let $c \in \ell^{1}\left(\mathbb{Z}_{+}^{3}\right)$ be $c=0$ and go to the final step. If not, let $j_{0}=\max \left\{j: a_{m-j, j} \neq 0\right\}$ and proceed to Step 2 if $j_{0}=0$ and to Step 3 if $j_{0} \neq 0$.

STEP 2. Let $c \in \ell^{1}\left(\mathbb{Z}_{+}^{3}\right)$ be such that $c_{m, 0,0}=a_{m, 0}, c_{i, j, k}=0$ elsewhere. Then $\left(d_{1}(c)\right)_{m, 0}=a_{m, 0}$ and clearly $\|c\|=p_{m}(f)$. Proceed to the final step.

STEP 3. It follows from (17) that there exists $k$ with $j_{0}>k>0$ such that $a_{m-j_{0}+k, j_{0}-k}$ is non-zero and of sign opposite to $a_{m-j_{0}, j_{0}}$. Let $k_{0}$ be the smallest such integer (so that $j_{0}-k_{0}$ is the largest possible) and let $\alpha=\operatorname{sgn}\left(a_{m-j_{0}, j_{0}}\right) \cdot \min \left\{\left|a_{m-j_{0}, j_{0}}\right|,\left|a_{m-j_{0}+k_{0}, j_{0}-k_{0}}\right|\right\}$. Let $c^{1} \in \ell^{1}\left(\mathbb{Z}_{+}^{3}\right)$ be such that $c_{m-j_{0}, k_{0}, j_{0}-k_{0}}^{1}=-\alpha, c_{i, j, k}^{1}=0$ for all other coordinates. Then $\left(d_{1}\left(c^{1}\right)\right)_{m-j_{0}, j_{0}}=\alpha,\left(d_{1}\left(c^{1}\right)\right)_{m-j_{0}+k_{0}, j_{0}-k_{0}}=\left(d_{1}\left(c^{1}\right)\right)_{m-k_{0}, k_{0}}=-\alpha$ and $\left(d_{1}\left(c^{1}\right)\right)_{j, k}=0$ otherwise. Clearly $\left\|c^{1}\right\|=|\alpha|$ and it is readily checked that $p_{m}\left(f-d_{1}\left(c^{1}\right)\right) \leq p_{m}(f)-\left\|c^{1}\right\|$. Note that this is the key part of the construction: $f$ has been modified by a coboundary $d_{1}\left(c^{1}\right)$ in such a way that $\left\|f-d_{1}\left(c^{1}\right)\right\|$ is less than $\|f\|$ by at least $\left\|c^{1}\right\|$.

If $\left(f-d_{1}\left(c^{1}\right)\right)_{m-j_{0}, j_{0}}=a_{m-j_{0}, j_{0}}-\alpha=0$, proceed to Step 4 letting $c=c^{1}$.

If $\left(f-d_{1}\left(c^{1}\right)\right)_{m-j_{0}, j_{0}}=a_{m-j_{0}, j_{0}}-\alpha \neq 0$, reapply the first part of this step to $f_{1}=f-d_{1}\left(c^{1}\right)$, noting that (17) holds for $f_{1}$. This yields a strictly larger value of $k_{0}$ and gives $c^{2}$, say, such that $0 \leq\left|\left(f-d_{1}\left(c^{1}+c^{2}\right)\right)_{m-j_{0}, j_{0}}\right|<$ 
$\left|\left(f-d_{1}\left(c^{1}\right)\right)_{m-j_{0}, j_{0}}\right|$ and $p_{m}\left(f-d_{1}\left(c^{1}+c^{2}\right)\right) \leq p_{m}(f)-\left\|c^{1}\right\|-\left\|c^{2}\right\|$. Iterate this until $c=c^{1}+c^{2}+\cdots+c^{n}$ (for some $n$ ) is such that $\left(f-d_{1}(c)\right)_{m-j_{0}, j_{0}}=0$. Note that the process must terminate as the $k_{0}$ are strictly increasing. It follows from the construction that $\left(f-d_{1}(c)\right)_{m-j, j}=0$ for $j_{0} \leq j \leq m$ and $p_{m}\left(f-d_{1}(c)\right) \leq p_{m}(f)-\|c\|$. Note also that $\|c\|=\left|a_{m-j, j}\right|$.

STEP 4. The iteration can now proceed to Step 1 with $f^{\prime}=f-d_{1}(c)$, and we get $c^{\prime}$, say, from Step 1, 2 or 3. If $c^{\prime}$ has come from Step 3, then $c^{\prime} \in \ell^{1}\left(\mathbb{Z}_{+}^{3}\right)$ is such that $\left(f^{\prime}-d_{1}\left(c^{\prime}\right)\right)_{m-j, j}=0$ for $j_{0}^{\prime} \leq j \leq m$ where $j_{0}^{\prime}<j_{0}$, with $p_{m}\left(f-d_{1}\left(c+c^{\prime}\right)\right) \leq p_{m}(f)-\left\|c+c^{\prime}\right\|$. This is iterated for $f^{\prime \prime}=f^{\prime}-d_{1}\left(c^{\prime}\right)$ to obtain $c^{\prime \prime}$, and so on, until the final step is reached, which is to say until the condition required in Step 1 or 2 is satisfied.

Final SteP. Let $b^{m}$ be the sum of $c, c^{\prime}$, etc., obtained in Step 1 or 2, as well as in Step 3. Note that we get at most one of those $c, c^{\prime}$, etc., from the application of Step 1 or 2, namely the last one obtained, and that all the previous ones arise from the (possibly repeated) application of Step 3. Then $\left(f-d_{1}\left(b^{m}\right)\right)_{m-j, j}=0$ for $0 \leq j \leq m$ and $0=p_{m}\left(f-d_{1}\left(b^{m}\right)\right) \leq p_{m}(f)-\left\|b^{m}\right\|$, so that $\left\|b^{m}\right\| \leq p_{m}(f)$, which completes the construction.

It follows from this proposition that, for each $n, \mathcal{H}_{n}(\mathcal{A}, \mathcal{A})$ is a Banach space and $B_{n}(\mathcal{A}, \mathcal{A})$ is closed. We can now state the following theorem.

Theorem 7.4. Let $\mathcal{A}=\ell^{1}\left(\mathbb{Z}_{+}\right)$. Then, for all $n$, the short exact sequences

$$
\begin{aligned}
& 0 \rightarrow B_{n}(\mathcal{A}, \mathcal{A}) \stackrel{j}{\rightarrow} Z_{n}(\mathcal{A}, \mathcal{A}) \stackrel{\sigma}{\rightarrow} \mathcal{H}_{n}(\mathcal{A}, \mathcal{A}) \rightarrow 0, \\
& 0 \rightarrow Z_{n+1}(\mathcal{A}, \mathcal{A}) \stackrel{i}{\rightarrow} C_{n+1}(\mathcal{A}, \mathcal{A}) \stackrel{\widetilde{d}}{\rightarrow} B_{n}(\mathcal{A}, \mathcal{A}) \rightarrow 0
\end{aligned}
$$

are split (as Banach spaces) and the $\mathcal{H}_{n}(\mathcal{A}, \mathcal{A}), C_{n}(\mathcal{A}, \mathcal{A}), Z_{n}(\mathcal{A}, \mathcal{A})$ and $B_{n}(\mathcal{A}, \mathcal{A})$ are strictly projective Banach spaces.

Proof. It is always true that $Z_{0}(\mathcal{A}, \mathcal{A})=C_{0}(\mathcal{A}, \mathcal{A})$ as the boundary map $d_{-1}$ is the zero map on $C_{0}(\mathcal{A}, \mathcal{A})$. Also, since $\mathcal{A}$ is commutative, $B_{0}(\mathcal{A}, \mathcal{A})$ is trivial and hence $\mathcal{H}_{0}(\mathcal{A}, \mathcal{A})=Z_{0}(\mathcal{A}, \mathcal{A})=C_{0}(\mathcal{A}, \mathcal{A})=\mathcal{A}$. By Proposition 7.3, for $n \geq 2, \mathcal{H}_{n}(\mathcal{A}, \mathcal{A})=\{0\}$ and therefore $Z_{n}(\mathcal{A}, \mathcal{A})=B_{n}(\mathcal{A}, \mathcal{A})$. Thus the sequences (18) and (19) are trivially split for $n=0$, and (18) splits for $n \geq 2$, as in these cases one term of the short exact sequence is 0 .

It is shown in Proposition 7.3 that, for $n=1$, the sequence (18) has the form

$$
0 \rightarrow B_{1}(\mathcal{A}, \mathcal{A}) \stackrel{j}{\rightarrow} \ell^{1}\left(\mathbb{Z}_{+}\right) \widehat{\otimes} \ell^{1}\left(\mathbb{Z}_{+}\right) \stackrel{q}{\rightarrow} \mathcal{I} \rightarrow 0 .
$$

Since this sequence is exact and $\operatorname{so} \operatorname{Im} j=\operatorname{Ker} q$, it is easy to check that it splits in $\mathcal{B}$ an with the following splitting continuous linear operators:

$$
p: \ell^{1}\left(\mathbb{Z}_{+}\right) \widehat{\otimes} \ell^{1}\left(\mathbb{Z}_{+}\right) \rightarrow B_{1}(\mathcal{A}, \mathcal{A}),
$$


given by

$$
p\left(z^{k} \otimes z^{l}\right)=\tilde{j}^{-1}\left[\left(z^{k} \otimes z^{l}\right)-\frac{l}{k+l}\left(1 \otimes z^{k+l}\right)\right],
$$

where $\widetilde{j}^{-1}$ is the inverse of the topological isomorphism $\widetilde{j}: B_{1}(\mathcal{A}, \mathcal{A}) \rightarrow \operatorname{Im} j$, and $t: \mathcal{I} \rightarrow \ell^{1}\left(\mathbb{Z}_{+}\right) \widehat{\otimes} \ell^{1}\left(\mathbb{Z}_{+}\right)$is given by $t\left(z^{k}\right)=1 \otimes z^{k}$. Therefore $B_{1}(\mathcal{A}, \mathcal{A})$ is complemented in $\ell^{1}\left(\mathbb{Z}_{+}^{2}\right)$ and hence is strictly projective.

This completes the basis for an induction showing that (19) splits for all values of $n$. To carry out this induction, we consider the following diagram:

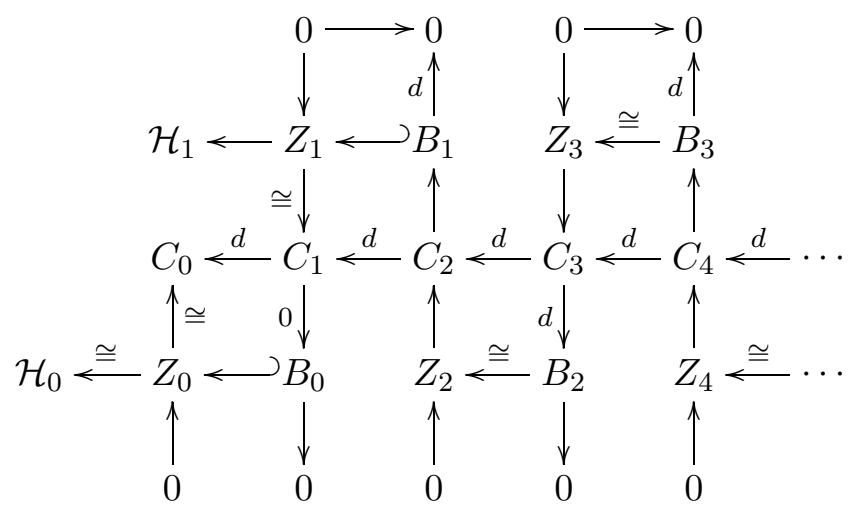

Note that as all of the $B_{n}(\mathcal{A}, \mathcal{A})$ and $\mathcal{H}_{n}(\mathcal{A}, \mathcal{A})$ are Banach spaces, this is a diagram of Banach spaces and continuous linear operators, where all the maps are boundaries, inclusions or quotients. The horizontal chain complex defines the homology $\mathcal{H}_{n}(\mathcal{A}, \mathcal{A})$, and the vertical parts of this diagram are short exact sequences of Banach spaces. It is clear that the diagram commutes.

Let us now proceed with the induction, assuming that (19) is split for $n$ and that $B_{n+1}(\mathcal{A}, \mathcal{A})$ is strictly projective. We have proved this for $n=0$, and we recall that $C_{n}(\mathcal{A}, \mathcal{A})=\ell^{1}\left(\mathbb{Z}_{+}^{n+1}\right)$ is a strictly projective Banach space.

As $B_{n+1}(\mathcal{A}, \mathcal{A})$ is strictly projective, (19) is split for $n+1$ and we have $C_{n+2}(\mathcal{A}, \mathcal{A}) \cong B_{n+1}(\mathcal{A}, \mathcal{A}) \oplus Z_{n+2}(\mathcal{A}, \mathcal{A})$. Therefore $Z_{n+2}(\mathcal{A}, \mathcal{A})$, being a direct summand of the strictly projective module $C_{n+2}(\mathcal{A}, \mathcal{A})$, is also strictly projective. As $\mathcal{H}_{n+2}(\mathcal{A}, \mathcal{A})=0$, we have $Z_{n+2}(\mathcal{A}, \mathcal{A})=B_{n+2}(\mathcal{A}, \mathcal{A})$ and $B_{n+2}(\mathcal{A}, \mathcal{A})$ is strictly projective. This completes the induction, and sequences (19) are split for all $n$. We note that we have also shown all the spaces to be strictly projective, which completes the proof of the theorem.

TheOREM 7.5. Let $\mathcal{A}=\ell^{1}\left(\mathbb{Z}_{+}\right)$. Then, up to topological isomorphism,

(1) $\mathcal{H}_{n}\left(\ell^{1}\left(\mathbb{Z}_{+}^{k}\right), \ell^{1}\left(\mathbb{Z}_{+}^{k}\right)\right)=0$ if $n>k$,

(2) $\mathcal{H}_{n}\left(\ell^{1}\left(\mathbb{Z}_{+}^{k}\right), \ell^{1}\left(\mathbb{Z}_{+}^{k}\right)\right)=\bigoplus^{\left(\begin{array}{l}k \\ n\end{array}\right)}\left(\mathcal{I}^{\widehat{\otimes}^{n}} \widehat{\otimes} \mathcal{A}^{\widehat{\otimes}^{k-n}}\right)$ if $n \leq k$,

(3) $\mathcal{H}^{n}\left(\ell^{1}\left(\mathbb{Z}_{+}^{k}\right), \ell^{1}\left(\mathbb{Z}_{+}^{k}\right)^{\prime}\right)=0$ if $n>k$, 
(4) $\mathcal{H}^{n}\left(\ell^{1}\left(\mathbb{Z}_{+}^{k}\right), \ell^{1}\left(\mathbb{Z}_{+}^{k}\right)^{\prime}\right)=\bigoplus^{\left(\begin{array}{l}k \\ n\end{array}\right)}\left[\left(\mathcal{I}^{\widehat{\otimes}^{n}} \widehat{\otimes} \mathcal{A}^{\widehat{\otimes}^{k-n}}\right)^{\prime}\right]$ if $n \leq k$,

where ( )' denotes the dual space.

Proof. For homology, Proposition 7.3 gives the result for $k=1$. Let $k>1$ and suppose that the result for homology holds for $k-1$. As $\ell^{1}\left(\mathbb{Z}_{+}^{k}\right)=\ell^{1}\left(\mathbb{Z}_{+}\right) \widehat{\otimes} \ell^{1}\left(\mathbb{Z}_{+}^{k-1}\right)$, we have

$$
\mathcal{H}_{n}\left(\ell^{1}\left(\mathbb{Z}_{+}^{k}\right), \ell^{1}\left(\mathbb{Z}_{+}^{k}\right)\right)=\mathcal{H}_{n}(\mathcal{A} \widehat{\otimes} \mathcal{B}, \mathcal{A} \widehat{\otimes} \mathcal{B}),
$$

where $\mathcal{B}=\ell^{1}\left(\mathbb{Z}_{+}^{k-1}\right)$.

Let us now consider the complex $C_{n}(\mathcal{A}, \mathcal{A})$. By Proposition 7.3, for all $n$, the $B_{n}(\mathcal{A}, \mathcal{A})$ are closed. By Theorem 7.4, for all $n$, the boundaries $B_{n}(\mathcal{A}, \mathcal{A})$ and the homology groups $\mathcal{H}_{n}(\mathcal{A}, \mathcal{A})$ of this complex are strictly projective and hence strictly flat. Also, it follows from the inductive hypothesis that, for all $n$, the $\mathcal{H}_{n}(\mathcal{B}, \mathcal{B})$ are Banach spaces and hence the $B_{n}(\mathcal{B}, \mathcal{B})$ are closed. We can therefore apply Corollary 6.4 to get

$$
\mathcal{H}_{n}(\mathcal{A} \widehat{\otimes} \mathcal{B}, \mathcal{A} \widehat{\otimes} \mathcal{B}) \cong \bigoplus_{m+q=n}\left[\mathcal{H}_{m}(\mathcal{A}, \mathcal{A}) \widehat{\otimes} \mathcal{H}_{q}(\mathcal{B}, \mathcal{B})\right] .
$$

The terms in this direct sum vanish for $m \geq 2$, and thus we only need to consider

$$
\left(\mathcal{H}_{0}(\mathcal{A}, \mathcal{A}) \widehat{\otimes} \mathcal{H}_{n}(\mathcal{B}, \mathcal{B})\right) \oplus\left(\mathcal{H}_{1}(\mathcal{A}, \mathcal{A}) \widehat{\otimes} \mathcal{H}_{n-1}(\mathcal{B}, \mathcal{B})\right) .
$$

If $n<k$ then from the induction hypothesis we get

$$
\begin{aligned}
\mathcal{H}_{n}\left(\ell^{1}\left(\mathbb{Z}_{+}^{k}\right), \ell^{1}\left(\mathbb{Z}_{+}^{k}\right)\right) \cong & \left(\mathcal{H}_{0}(\mathcal{A}, \mathcal{A}) \widehat{\otimes} \mathcal{H}_{n}(\mathcal{B}, \mathcal{B})\right) \oplus\left(\mathcal{H}_{1}(\mathcal{A}, \mathcal{A}) \widehat{\otimes} \mathcal{H}_{n-1}(\mathcal{B}, \mathcal{B})\right) \\
\cong & \left(\mathcal{A} \widehat{\otimes}\left(\bigoplus^{\left(\begin{array}{c}
k-1 \\
n
\end{array}\right)} \mathcal{I}^{\widehat{\otimes}^{n}} \widehat{\otimes} \mathcal{A}^{\widehat{\otimes}^{k-1-n}}\right)\right) \\
& \oplus\left(\mathcal{I} \widehat{\otimes}\left(\bigoplus^{\left(\begin{array}{c}
k-1 \\
n-1
\end{array}\right)} \mathcal{I}^{\widehat{\otimes}^{n-1}} \widehat{\otimes} \mathcal{A}^{\widehat{\otimes}^{k-n}}\right)\right) \\
\cong & \bigoplus^{\left(\begin{array}{l}
k \\
n
\end{array}\right)}\left(\mathcal{I}^{\widehat{\otimes}^{n}} \widehat{\otimes} \mathcal{A}^{\widehat{\otimes}^{k-n}}\right) .
\end{aligned}
$$

The other cases easily follow from the induction hypothesis: if $n>k$, then $\mathcal{H}_{n}(\mathcal{B}, \mathcal{B})$ and $\mathcal{H}_{n-1}(\mathcal{B}, \mathcal{B})$ both vanish, while if $n=k, \mathcal{H}_{n}(\mathcal{B}, \mathcal{B})=0$ and $\mathcal{H}_{n-1}(\mathcal{B}, \mathcal{B})=\mathcal{I}^{\widehat{\otimes}^{n-1}}$, and the result follows immediately, proving (1) and (2). Statements (3) and (4) now follow directly from Corollary 6.4.

Acknowledgments. The first author thanks the University of Newcastle upon Tyne for its kind hospitality while this paper was being written.

\section{References}

[1] C. Bănică and O. Stănăşilă, Méthodes algébriques dans la théorie globale des espaces complexes, Gauthier-Villars, Paris, 1977. 
[2] J. Brodzki and Z. A. Lykova, Excision in cyclic type homology of Fréchet algebras, Bull. London Math. Soc. 33 (2001), 283-291.

[3] J. Cigler, V. Losert and P. W. Michor, Banach Modules and Functors on Categories of Banach Spaces, Dekker, New York, 1979.

[4] R. E. Edwards, Functional Analysis. Theory and Applications, Holt, Rinehart and Winston, New York, 1965.

[5] J. Eschmeier and M. Putinar, Spectral Decompositions and Analytic Sheaves, London Math. Soc. Monogr. 10, Clarendon Press, Oxford, 1996.

[6] F. Gourdeau, B. E. Johnson and M. C. White, The cyclic and simplicial cohomology of $l^{1}(\mathbb{N})$, Trans. Amer. Math. Soc., to appear.

[7] A. Ya. Helemskiŭ, The Homology of Banach and Topological Algebras, Kluwer, Dordrecht, 1989.

[8] B. E. Johnson, Cohomology in Banach algebras, Mem. Amer. Math. Soc. 127 (1972).

[9] G. Köthe, Hebbare lokalkonvexe Räume, Math. Ann. 165 (1966), 181-195.

[10] J. Lindenstrauss and L. Tzafriri, Classical Banach Spaces I, Springer, New York, 1977.

[11] Z. A. Lykova, Excision in Banach simplicial and cyclic cohomology, Proc. Edinburgh Math. Soc. (2) 41 (1998), 411-427.

[12] - Cyclic-type cohomology of strict inductive limits of Fréchet algebras, preprint, 2003.

[13] S. MacLane, Homology, Springer, Berlin, and Academic Press, New York, 1963.

[14] F. Prosmans, Quasi-Abelian homological algebra, Mémoire de DEA, Université Paris 13, juin 1995; http://users.belgacom.net/fprosmans

[15] M. A. Rieffel, Induced Banach representations of Banach algebras and locally compact groups, J. Funct. Anal. 1 (1967), 443-491.

[16] F. Trèves, Topological Vector Spaces, Distributions and Kernels, Academic Press, New York, 1967.

Département de Mathématiques

Université Laval

Cité Universitaire (Québec)

G1K 7P4, Canada

E-mail: Frederic.Gourdeau@mat.ulaval.ca
School of Mathematics and Statistics University of Newcastle upon Tyne Newcastle upon Tyne, NE1 7RU, UK E-mail: Z.A.Lykova@ncl.ac.uk Michael.White@ncl.ac.uk

Received April 18, 2003

Revised version August 30, 2004 